\title{
Specification of a Foxj1-Dependent Lineage in the Forebrain Is Required for Embryonic-to-Postnatal Transition of Neurogenesis in the Olfactory Bulb
}

\author{
Benoit V. Jacquet, ${ }^{1}$ Nagendran Muthusamy, ${ }^{1}$ Laura J. Sommerville, ${ }^{1}$ Guanxi Xiao, ${ }^{1}$ Huixuan Liang, ${ }^{1}$ Yong Zhang, ${ }^{2}$ \\ Michael J. Holtzman, ${ }^{2}$ and $\mathrm{H}$. Troy Ghashghaei ${ }^{1}$ \\ ${ }^{1}$ Center for Comparative Medicine and Translational Research, Department of Molecular Biomedical Sciences, College of Veterinary Medicine, North \\ Carolina State University, Raleigh, North Carolina 27606, and ${ }^{2}$ Pulmonary and Critical Care Medicine, Department of Internal Medicine, Washington \\ University School of Medicine, St Louis, Missouri 63110
}

Establishment of a neural stem cell niche in the postnatal subependymal zone (SEZ) and the rostral migratory stream (RMS) is required for postnatal and adult neurogenesis in the olfactory bulbs (OB). We report the discovery of a cellular lineage in the SEZ-RMS-OB continuum, the specification of which is dependent on the expression of the forkhead transcription factor Foxj1 in mice. Spatially and temporally restricted Foxj1 + neuronal progenitors emerge during embryonic periods, surge during perinatal development, and are active only for the first few postnatal weeks. We show that the development of the unique Foxj1-derived lineage is dependent on Foxj1 expression and is required for overall postnatal neurogenesis in the OB. Strikingly, the production of neurons from Foxj1 + progenitors significantly declines after the early postnatal weeks, but Foxj1-derived neurons in the OB persist during adult periods. For the first time, our study identifies the time- and region-specific activity of a perinatal progenitor domain that is required for transition and progression of $\mathrm{OB}$ neurogenesis from the embryonic-to-postnatal periods.

\section{Introduction}

Neuronal specification is dependent on molecular signaling in the embryonic neuroepithelium. In mice, the neuroepithelium expands during early CNS development and is the source of a remarkably diverse population of embryonic neural stem cells and progenitors until early postnatal periods (Guillemot, 2007; Pinto and Götz, 2007; Kriegstein and Alvarez-Buylla, 2009; Okano and Temple, 2009). The temporal and spatial induction of the heterogeneous population of neural progenitors is further controlled by organizer and patterning centers that secrete and generate gradients of morphogens, cytokines, and growth factors throughout the developing brain (Guillemot, 2005; O'Leary et al., 2007; Hoch et al., 2009; Gaspard and Vanderhaeghen, 2010).

Progenitors in the embryonic neuroepithelium differentiate into astrocytes and ependymal cells that line the cerebral ventricles around the time of birth (Schmechel and Rakic, 1979; Merkle

Received Jan. 10, 2011; revised May 11, 2011; accepted May 16, 2011.

Author contributions: H.T.G. designed research; B.V.J., N.M., L.J.S., G.X., H.L., and H.T.G. performed research; Y.Z. and M.J.H. contributed unpublished reagents/analytic tools; B.V.J. and H.T.G. analyzed data; H.T.G. wrote the paper.

This work was supported by National Institutes of Health Grant R01NS062182, a grant from the American Federation for Aging Research, and institutional funds to H.T.G. Sooyoung Franck, Samina Ali, and Alice Harvey provided excellent technical support. We thank Drs. Steven Brody (Washington University), Robert Hevner (University of Washington), Eric Tucker (West Virginia University), Kenny Campbell (Cincinnati Children's Hospital), David Eisenstat (Manitoba Institute of Cell Biology), Larry Ostrowski and John Olsen (UNC-Chapel Hill), Brigid Hogan (Duke University), and Gord Fishell (New York University) for constructs, antibodies, and mice.

Correspondence should be addressed to H. Troy Ghashghaei, Center for Comparative Medicine and Translational Research, Department of Molecular Biomedical Sciences, College of Veterinary Medicine, North Carolina State University, 4700 Hillsborough Street, Raleigh, NC 27606. E-mail: Troy_Ghashghaei@ncsu.edu.

DOI:10.1523/JNEUROSCI.0171-11.2011

Copyright $\odot 2011$ the authors $\quad 0270-6474 / 11 / 319368-15 \$ 15.00 / 0$ et al., 2004; Spassky et al., 2005; Jacquet et al., 2009b). During the same period most progenitor domains become dormant, but discrete sets remain developmentally active and generate neurons in the olfactory bulbs (OBs) throughout life (Kriegstein and Alvarez-Buylla, 2009). Progenitors for adult OB neurons are situated within the subependymal zone of the neostriatum (SEZ) and in the rostral migratory stream (RMS), which are remnants of the embryonic olfactory ventricles (Doetsch et al., 1997). These neurogenic tissues are derived from embryonic progenitor domains that generate most of the $\mathrm{OB}$ neurons during development.

OB histogenesis involves a coordinated development of olfactory receptor axons from the nasal epithelium and central neurogenesis within the telencephalon. Although the development of the peripheral olfactory receptors has been extensively studied (for review, see Sakano, 2010), the generation of central OB neurons has received far less attention (for review, see Lledo et al., 2008). Olfactory bulb neurons are generated in the embryonic lateral ganglionic eminence (LGE) and its rostral extension in the olfactory ventricular zone (OVZ) (Kriegstein and Alvarez-Buylla, 2009). During early postnatal development, the olfactory ventricle collapses and the dorsal and ventral aspects of the OVZ fuse to form the RMS, while the ventricular and subventricular zones of the LGE transform into the SEZ. The timing of these transformations coincides with the transition of embryonic-to-postnatal neurogenesis and differentiation in the OB-specific stem cell niches. Progenitors in the LGE and OVZ reside in a radial glialrich niche, while the adult stem cell niches in the SEZ and RMS largely consist of ependymal cells, astrocytes, and vascular endo- 
thelial cells. Adult "niche cells" are thought to help regulate cellular proliferation, migration, and differentiation that is required for neurogenesis in the $\mathrm{OB}$, but their precise roles remain largely unknown (Shen et al., 2008; Tavazoie et al., 2008; Kokovay et al., 2010).

We recently showed that the differentiation of radial glial cells into ependymal cells and a small population of astrocytes in the adult-stage stem-cell niche requires time- and cell-specific expression of the Forkhead transcription factor Foxj1 (Jacquet et al., 2009b). Foxj1 is required for multiple developmental processes in ependymal cell differentiation, including a canonical role in genesis of motile cilia and establishment of planar and baso-apical polarity. Here, we report the unexpected discovery of a Foxj1-dependent cellular lineage that stretches from the SEZ, through the RMS and into the OB, revealing a novel cell-specific regulatory center embedded within this neurogenic tissue in the postnatal and adult rostral forebrain.

\section{Materials and Methods}

Animals. Animals were housed and used in compliance with Institutional Animal Care and Use Committee and North Carolina State University regulations. Foxj $1^{-1-}$ EGFP mice were obtained by crossing Foxj $1^{\text {EGFP }}$ mice with Foxj $1^{+/-}$mice (Jacquet et al., 2009b). Foxj $1^{-1-E G F P}$ mice and their littermate controls were killed at multiple developmental stages. For fixed analysis of tissue, animals were killed by Avertin overdose $(7.5 \mathrm{mg} / \mathrm{g}$ body weight) via intraperitoneal injections, followed by transcardial perfusion with $4 \%$ paraformaldehyde in $0.1 \mathrm{~m}$ PBS, pH 7.4. Brains were removed and postfixed for $24 \mathrm{~h}$ and then sectioned at $50 \mu \mathrm{m}$ on a vibratome (Leica VT 1000 S). Foxj1-independent hydrocephalic models (Kaolin-induced and Dnaic ${ }^{-1-}$ mice) were described previously (Jacquet et al., 2009b). A minimum of three animals of either sex and with the appropriate genotypes were used in each experiment.

In vivo fate mapping. In vivo fate mapping experiments were conducted by crossing Foxj1 ${ }^{\text {Cre::ER }}$ (Rawlins et al., 2007) and Nestin ${ }^{\text {Cre::ER }}$ mice (Balordi and Fishell, 2007a) with reporter B6;129S4-Gt(ROSA) 26Sor ${ }^{\text {tm1Sor }} / \mathrm{J}$ mice (Jackson Laboratory Stock\# 003309), B6;129S6Gt(ROSA) 26Sortm14(CAG-tdTomato)Hze (Jackson Laboratory Stock\# 007908) mice, and pCAG-floxed STOP-EGFP mice (Generous gift from Dr. K. Campbell, Cincinnati Children's Hospital, Cincinnati, OH). Four milligrams of tamoxifen (TAM; dissolved at $20 \mathrm{mg} / \mathrm{ml}$ in corn oil-United States Biochemical cat\# 13851) was administered as a single intraperitoneal injection to the nursing dam for postnatal day 0 (P0) inductions. P21 and P60 mice were TAM induced with single $2 \mathrm{mg}$ i.p. injections. Constitutive labeling of Foxj1 + cells and their derivatives was achieved by crossing Foxj ${ }^{\text {Cre }}$ mice (Zhang et al., 2007) to the same reporter lines as listed above. A word of caution for the use of Foxj $1^{\text {Cre }}$ or Foxj $1^{\text {Cre::ER mice: }}$ carrying floxed alleles together with these cre lines in breeders results in spontaneous recombination in germ cells and generation of mosaic animals that contain the floxed-out gene throughout the body. We find this to occur more often in male breeders, which we presume is due to high levels of cre expression under the Foxj1 promoter in sperm cells. To obtain cell-specific induction of cre it is critical that each breeding pair carries either the cre allele or the floxed allele, but not both.

BrdU administration. 5-Bromo-2'-deoxyuridine (BrdU, Aldrich $\# 85811-5 \mathrm{G}$ ) was diluted in saline at a concentration of $10 \mathrm{mg} / \mathrm{ml}$ and single pulses were administered intraperitoneally at $100 \mu \mathrm{g} / \mathrm{g}$ body weight. Mice were perfused $1 \mathrm{~h}$ after BrdU administration for acute analysis of proliferating progenitors in the LGE, OVZ, SEZ, and RMS. For birth-dating experiments, timed-pregnant females were administered $\mathrm{BrdU}(50 \mu \mathrm{g} / \mathrm{g}$ body weight) three times on embryonic days 12.5 and 16.5 (E12.5 and E16.5). For postnatal birth-dating, P0 and P14 mice were administered BrdU (intraperitoneally) three times over a $12 \mathrm{~h}$ period. All mice were killed at P21.

Tissue processing and immunohistochemistry. Brain sections were blocked for $1 \mathrm{~h}$ at room temperature in $10 \%$ goat serum with $1 \%$ Triton X (Sigma, Cat. \# S26-36-23) dissolved in PBS, followed by overnight incubation with primary antibodies at $4^{\circ} \mathrm{C}$. For Ki67 staining, epitope retrieval was achieved by washing the sections three times with boiling 10 mm sodium citrate, $\mathrm{pH} 8.5$, for $15 \mathrm{~s}$ before blocking. For visualization of BrdU labeling, sections were treated with $2 \mathrm{~N} \mathrm{HCl}$ for $15 \mathrm{~min}$, followed by three $5 \mathrm{~min}$ washes in $0.1 \mathrm{~m}$ sodium borate, $\mathrm{pH}$ 8.6, before blocking. Secondary antibodies used for visualization were generally made in goat and were conjugated to Alexa 488, Cy3, and Alexa 647 (all diluted 1:1000, $1 \mathrm{~h}$ incubation at room temperature). Antibodies used were as follows: rabbit anti-Dlx2 (D. Eisenstat, Manitoba Institute of Cell Biology, Winnipeg, MB, Canada; 1:1000), mouse anti-GFAP (Millipore; 1:1000), rabbit antiGFAP (DakoCytomation; 1:1000), chicken anti-GFP (Abcam; 1:2000), rabbit anti-Gsx2 (K. Campbell; 1:4000), rabbit anti-S100ß (Sigma; 1:1000), rabbit anti-phospho-histone3 (Millipore; 1:500), mouse anti-Tuj1 (Covance; 1:200), rabbit anti-NG2 (LifeSpan Biosciences; 1:500), rabbit antiKi67 (Vision Biosystems; 1:250), mouse anti-calbindin (Abcam; 1:500), rabbit anti-calbindin (Abcam; 1:500), rabbit anti-calretinin (Abcam; 1:500), rabbit anti-tyrosine hydroxylase (Millipore; 1:500), mouse anti-NeuN (Millipore; 1:1000), rabbit anti-Pax6 (Millipore; 1:500), mouse anti-NCAM (Abcam), chicken anti- $\beta$-galactosidase (Abcam; 1:1000), guinea pig anti-Dcx (Millipore; $1: 1000$ ), mouse anti-BrdU (BD Bioscience; 13:1000), rabbit anti-Tbr1 (Millipore; 1:1000; and gift from Dr. Robert Hevner, University of Washington, Seattle, WA), chicken anti-Tbr1 (Millipore; 1:1000), rabbit anti-Tbr2 (Millipore; 1:1000), mouse anti-Foxj1 (eBioscience; 1:1000), rabbit anti-RFP (Abcam; 1:1000), rabbit anti-GABA (Millipore; 1:1000), rabbit anti-GAD (Sigma; 1:1000), and Cy3-conjugated Nissl stain (Invitrogen; 1:1000).

Neurosphere cultures. P0 and P21 Foxj $1^{+/+E G F P}$ and Foxj $1^{-/-E G F P}$ brains were harvested and the SEZ and RMS were microdissected in HBSS supplemented with 2.5 mM HEPES, pH 7.4, 30 mM D-glucose, $1 \mathrm{~mm}$ $\mathrm{CaCl}_{2}, 1 \mathrm{~mm} \mathrm{MgSO}_{4}$, and $4 \mathrm{~mm} \mathrm{NaHCO}_{3}$. Cultures were obtained from microdissected tissues in two groups, in one set Foxj1 $1^{+/+E G F P}$ and Foxj1 $1^{-1-E G F P}$ cells were sorted using a DakoCytomation MoFlo, in another set cells were isolated and cultured unsorted. Both groups of cells were cultured at $37^{\circ} \mathrm{C} / 5 \% \mathrm{CO}_{2}$ in neurobasal medium (Invitrogen \#21103-049) supplemented with hEGF (Invitrogen \#13247-051) and bFGF (Invitrogen \#13256-029), as previously described (Jacquet et al., $2009 \mathrm{~b}$ ). Neurospheres were passaged every $5 \mathrm{~d}$ by mechanical dissociation into a single cell suspension before reculturing. Cultures were supplemented with growth factors every $2 \mathrm{~d}$.

Conditioned medium $(\mathrm{CM})$ used to rescue the FoxJ1 ${ }^{-1-}$ neurospheres was collected from wild-type neurosphere cultures $4 \mathrm{~d}$ following the appearance of neurospheres. Floating cells and neurospheres were spun down to ensure absence of cellular contamination in the wild-type CM.

Neurospheres were differentiated on chambered glass slides coated with laminin (Sigma \#L2020) and poly-D-lysine (BD Falcon \#354417) in neurobasal medium devoid of growth factors for $10 \mathrm{~d}$. Differentiated cultures were then fixed in ice-cold $4 \%$ paraformaldehyde and immunostained as described in Tissue processing and immunohistochemistry.

Constructs and in vivo electroporation. The Foxj $1 \mathrm{cDNA}$ was purchased from ATCC and subcloned into the pGem vector (Invitrogen). The Foxj1 open reading frame (ORF) was amplified from the pGem vector with primers containing flanking XhoI and BamHI restriction sites and inserted into a vector containing a $1.5 \mathrm{~kb}$ fragment of the Foxj1 promoter (Gift from Larry Ostrowski and John Olsen, University of North Carolina at Chapel Hill, Chapel Hill, NC). The resultant pFoxj1:Foxj1ORF cassette was then amplified and inserted into a $p C A G: I R E S:$ tdTomato expression vector replacing the pCAG promoter (Gift from Dr. E. Tucker, West Virginia University, Morgantown, WV; final vector denoted as pFoxj1:Foxj1ORF:IRES:tdTomato). The reasoning behind driving the expression of Foxj1ORF downstream of its own promoter was that multiple attempts to express the Foxj1 under the pCAG promoter resulted in massive cell death in targeted wild-type and Foxj1-null neural progenitors. P0 wild-type and Foxj1-null mice on the Foxj1 ${ }^{\text {EGFP }}$ background were rapidly anesthetized by hypothermia followed by intraventricular injection of the plasmids $(5 \mu \mathrm{l}$ of $1 \mu \mathrm{g} / \mu \mathrm{l}$ construct in sterile water). The heads of injected mice were then electroporated using a BTX electroporation apparatus ( 4 unipolar pulses, $150 \mathrm{~V}, 40 \mathrm{~ms}$ per pulse, 950 $\mathrm{ms}$ intervals). Mice were then revived rapidly under a heating lamp and placed back with the dam for $2 \mathrm{~h}$. The brains of electroporated pups were 
A

E11.5
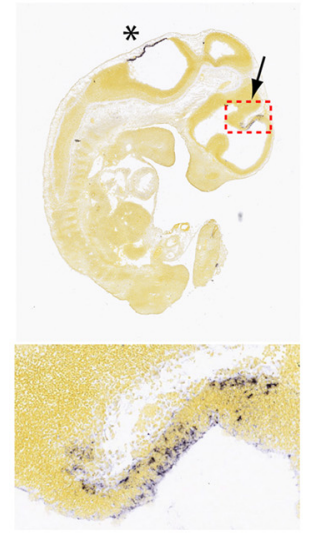

B
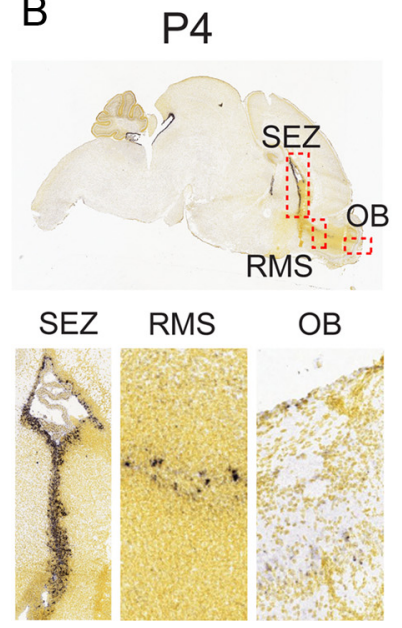

E13.5

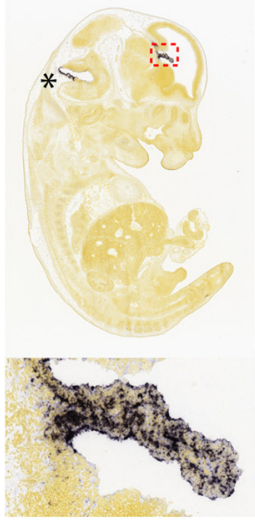

E15.5

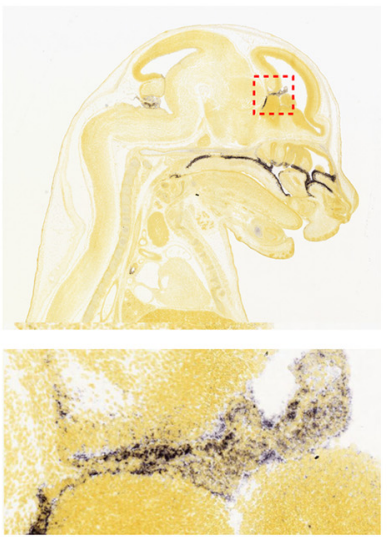

E18.5
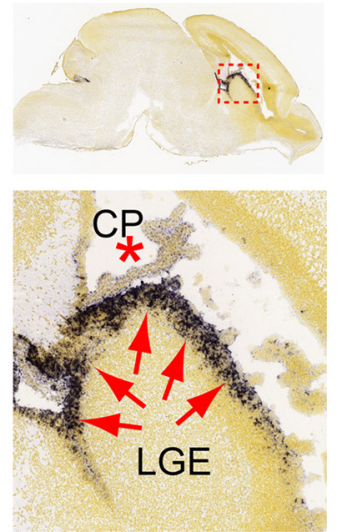

P14

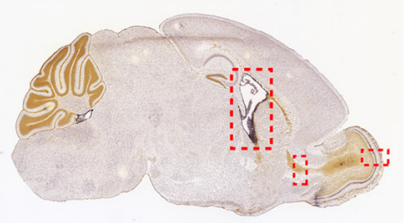

SEZ

RMS

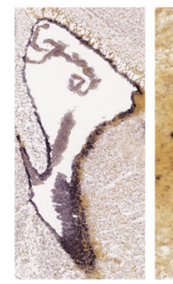

OB

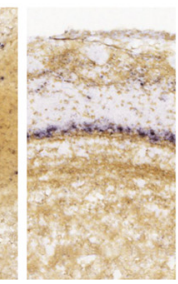

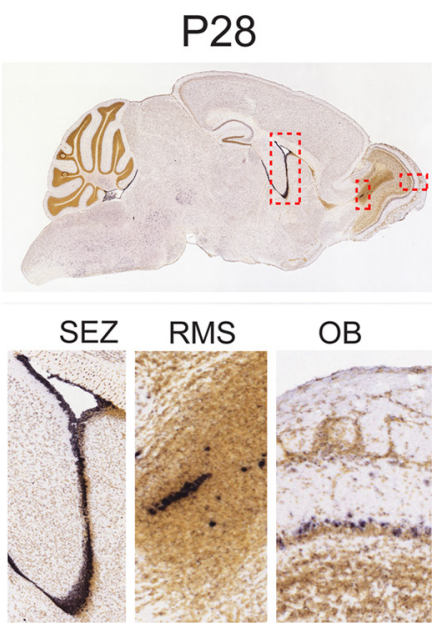

Figure 1. Developmental expression of Foxj1 in the brain. All photomicrographs are in situ hybridization panels revealing Foxj1 expression, obtained from the Developing Brain Module at the Allen Brain Atlas website (http://developingmouse.brain-map.org/). Dark blue/purple color corresponds to Foxj1 mRNA. A, Foxj1 expression in the embryonic brain. High-power images are magnified from regions demarcated by red dotted boxes in the low-power images above them. Black asterisks at E11.5 and E13.5 indicate Foxj1 expression in the floor plate of the caudal rhombomeres. Red arrows at E18.5 indicate increased Foxj1 expression in the ventricular wall of the LGE. Red asterisk shows downregulated expression in the choroid plexus at this stage. $\boldsymbol{B}$, Foxj 1 expression during postnatal development. Boxed areas in low-power images are labeled accordingly below each stage.

then harvested and cells were obtained for neurosphere and the differentiation assays, as described above.

Data quantification and analysis. Stereological volume estimations, absolute cell number estimations, cell density estimations, and normalization of data were conducted as previously described (Ghashghaei et al., 2007). Percentage of tdTomato + cells positive for various markers was calculated by counting 100 tdTomato + cells in different layers of the OB in each of three different animals at multiple ages. Error was calculated as the SEM and significance was determined using Student's $t$ test.

For quantification of in vitro data, the number of neurospheres and the rate of sphere growth were calculated by obtaining five random images from each culture-well every $24 \mathrm{~h}$, and measurements were conducted in ImageJ. The rate of growth was calculated as an average increase in diameter within individual spheres per day.

\section{Results}

Timing of Foxj1 expression in a restricted forebrain progenitor domain coincides with interruption of histogenesis in the Foxj $1^{-/-}$olfactory bulbs

We recently showed that Foxj1 is expressed in ependymal cells, as well as in a small subset of astrocytes and immature glia in the adult SEZ and RMS (Jacquet et al., 2009b). To confirm and validate those findings, we analyzed the temporal and spatial fore- brain expression pattern of Foxj1 during embryonic and early postnatal development using in situ hybridization panels from the Allen Brain Atlas. At E11.5, Foxj1 expression was confined to the primordial choroid plexis, and within the floor plate of hindbrain rhombomeres (Fig. 1A). From E13.5 to E15.5, Foxj1 expression became more pronounced in the choroid plexus (Fig. 1A). However, only sporadic mRNA (Fig. 1A) (E11.5-E15.5) or antibody staining (data not shown) was observed in the dorsolateral LGE where robust Foxj1-promoter activity can be detected in Foxj $1^{\text {EGFP }}$ reporter mice, as described previously (Jacquet et al., 2009b).

Between E15.5 and E18.5, a thin layer of cells in the ventricular zone of the LGE, corresponding to the region that exhibited promoter activity in Foxj $1^{\text {EGFP }}$ brains of younger embryos (Jacquet et al., 2009b), began to express Foxj1 transcript (Fig. $1 A$, red arrows in E18.5). By E18.5, scattered cells in the OVZ expressed Foxj1, whereas Foxj1 expression visibly declined in the choroid plexus (Fig. 1A, red asterisk in E18.5). The late embryonic expression pattern of Foxj1 in the dorsolateral LGE was confirmed with antibody staining, although protein distribution was patchy (data not shown) compared with the transcript expression revealed in panels from the Allen Brain Atlas. 

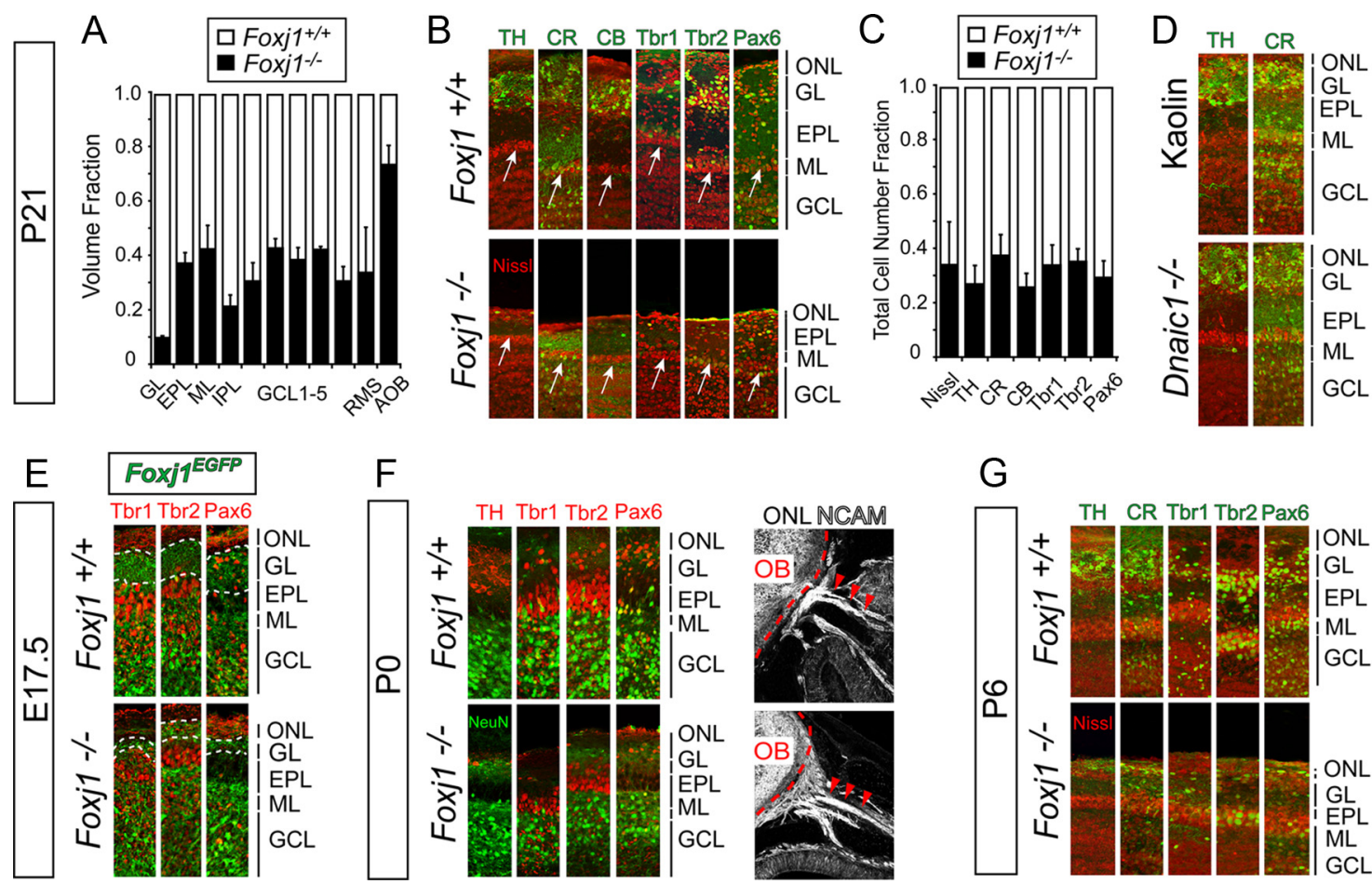

Figure 2. Disrupted perinatal development in the Foxj $1^{-1-} \mathrm{OB}$. A, Stereological volume estimations of different layers of the Foxj $1^{-/-} \mathrm{OB}$ at P21. Data were normalized and presented as fractions of Foxj $1^{-1-}$ volumes over wild-type volumes $(n=3)$. $\boldsymbol{B}$, Immunofluorescence staining of OB layers in the P21 Foxj $1^{-1-}$ and Foxj ${ }^{+/+}$mice. Staining reveals the absence of a GL from the Foxj $1^{-I-}$ OB. The mitral cell layer is identified for reference (arrows). TH, CB, CR, Tbr1 and Tbr2, and Pax6 immunoreactivities delineate cellular deficits in the P21 Foxj ${ }^{-1-}$ OB. (Layers of the OB: ONL, GL, EPL, ML, GCL.) C, Bar chart indicates significant reductions in estimated absolute number of all cells (Nissl) and specific cell types in the P21 Foxj $1^{-1-}$ OB. (Data are normalized; mean \pm SEM. $n=3 ; p<0.001)$. D, Intact OB lamination revealed by $\mathrm{TH}_{\mathrm{H}}$ and $\left(\mathrm{R}\right.$ staining in the brains of a genetic (Dnaic ${ }^{-l-}$ ) and a kaolin-induced mouse model of hydrocephalus. $\boldsymbol{E}$, Immunofluorescence staining for markers in the E17.5 OB. The GL, demarcated by the dashed line, is significantly acellular in the Foxj ${ }^{-1-}$ OB atE17.5.F, By P0, neuronal populations remain visibly reduced (NeuN, green) in the Foxj $1^{-1-}$ OB. Tbr1-, Tbr2-, and Pax6-expressing neurons fail to occupy the Foxj $1^{-1-} \mathrm{GL}$ as they do in wild-type mice. In sagittal sections of whole heads obtained from PO Foxj ${ }^{-1-}$ and Foxj ${ }^{+/+}$pups, olfactory axons positive for the NCAM were clearly present in the PO Foxj $1^{-1-}$ ONL (red arrowheads) and appeared to sprout around the OB. G, Immunostaining for neuronal markers in the P6 Foxj $1^{-/-}$and Foxj $1^{+/+}$OB.

During early postnatal development (between P4 and P6), Foxj1 expression expanded and became more robust beyond the confined dorsolateral LGE domain and included the entire rostrocaudal and mediolateral extents of the ventricular walls in the forebrain (Fig. 1B). This pattern confirmed our previous report that a perinatal upsurge in Foxj1 expression cell-autonomously regulates differentiation of ependymal cells, including the genesis of motile cilia after birth (Jacquet et al., 2009b). However, radial glial cells within the dorsolateral domain of the LGE, which began their Foxj1 expression more than a week earlier, differentiate into ependymal cells late (between P6 and P10) relative to domains outside the LGE (between P4 and P6) (Spassky et al., 2005; Kuo et al., 2006; Jacquet et al., 2009b). In essence, LGE and OVZ cells that express Foxj1 first in the rostral forebrain are the last to mature into ependymal cells.

At later postnatal time points (P14-P28), Foxj1 expression in the rostral forebrain was restricted to the SEZ and a narrow band of cells within the RMS and the core of the OB, as well as in cells within the mitral cell layer (Fig. $1 B$ ). While the pattern of antibody staining overlapped with mRNA expression in SEZ and RMS, we failed to confirm Foxj1 protein expression in the mitral cell layer (data not shown). The mapping of Foxj1 promoter activity (Jacquet et al., 2009b) together with transcript and protein expression led us to hypothesize that the perinatal expression of Foxj1 in the LGE and OVZ may have alternative functions to Foxjl's canonical role in the induction of differentiation and genesis of motile cilia in ependymal cells (Jacquet et al., 2009b).
One clue for a potential alternative function of perinatal Foxj1 expression came from the finding that the volume of the P21 Foxj1 ${ }^{-1-}$ OB was significantly smaller than the wild-type OB (Jacquet et al., 2009b). Stereological volume estimations revealed that Foxj1 deletion resulted in highly significant reductions in the volume of every $\mathrm{OB}$ layer at $\mathrm{P} 21$, and the most profound defect was the absence of the glomerular layer (GL) (Fig. 2A). Cellular enumerations indicated massive depletion of neurons expressing the dopaminergic enzyme tyrosine hydroxylase (TH) and the calcium binding proteins calbindin (CB) and calretinin (CR) (Fig. $2 \mathrm{~B}$ ). Moreover, neurons expressing the transcription factors paired homeobox domain 6 (Pax6) and T-brain homeobox domain factors 1 and 2 (Tbr1 and Tbr2), which label dopaminergic and glutamatergic neurons in the GL (Hack et al., 2005; Brill et al., 2009), were nearly abolished along with the missing GL (Fig. 2B). Stereological estimation of absolute numbers of various neuronal markers in the OB revealed significant decreases in the population of all identified neuronal types in the Foxj1 $1^{-1-}$ OB when compared with age-matched wild types (Fig. 2C). These defects resulted in a significant reduction in the overall size of the Foxj1 ${ }^{-1-} \mathrm{OB}$, which at $\mathrm{P} 21$ resembled a $\mathrm{P} 0$ wild-type OB.

To ensure that the decrease in cellular numbers in the P21 Foxj1 ${ }^{-1-}$ OB was independent of the hydrocephalus that gradually develops in these mice (Jacquet et al., 2009b), we assessed OB lamination in a genetic $\left(\right.$ Dnaic $\left.^{-1-}\right)$ and a kaolin-induced model of hydrocephalus (Jacquet et al., 2009b). The presence of the GL revealed that $\mathrm{OB}$ histogenesis remains intact and was similar to wild types at multiple developmental time points up to 

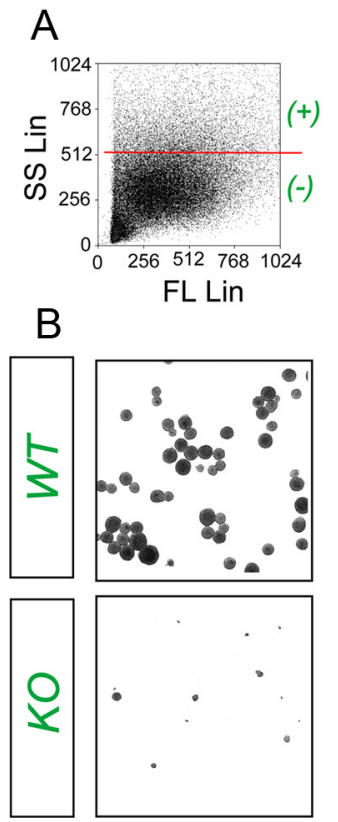

C
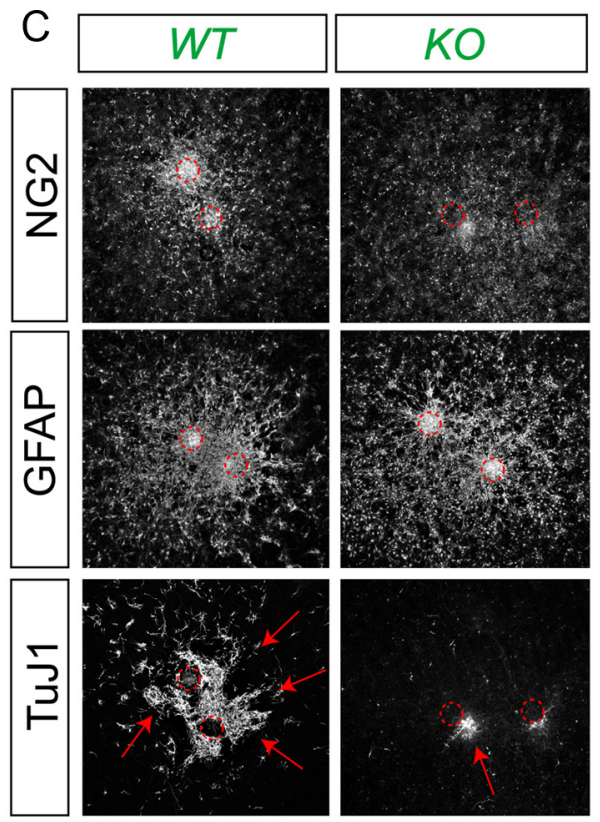
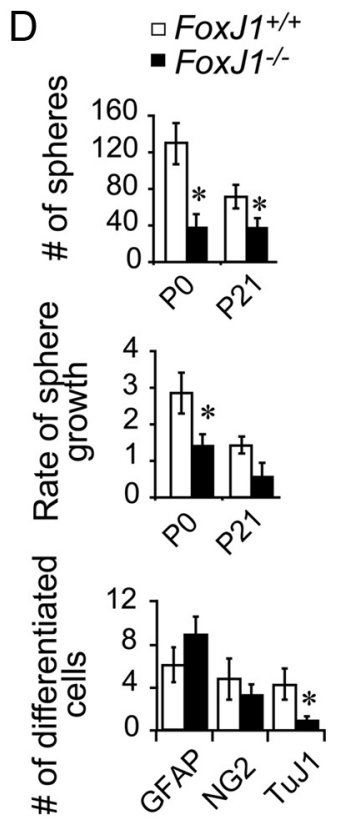

Figure 3. Proliferative capacity and differentiation potential of Foxj $1^{-1-}$ progenitors in vitro. $A$, Sorting of P0 and P21 Foxj ${ }^{\text {EGFP }}$ progenitor cells into EGFP + and EGFP-negative populations by FACS. B, Representative photomicrographs of Foxj $1^{+/+E G F P}$ and Foxj $1^{-1-\text { EGFP }}$ neurosphere cultures. Foxj $1^{-1-E G F P}$ progenitors generated significantly fewer neurospheres than Foxj $1^{+/+E G F P}$ cells. The few Foxj $1^{-I-}$ neurospheres grew significantly slower than control neurospheres. C, Neurospheres were plated on laminin/poly-D-lysine (dotted circles). Cells derived from Foxj ${ }^{-I-}$ spheres were highly gliogenic (GFAP and NG2 positive), but have impaired neurogenic potential (Tuj1, arrows). D, Quantification of the number, rate, and differentiation potential of Foxj ${ }^{-1-E G F P}+$ and Foxj $1^{+/+E G F P}+$ neurospheres. Numbers of cells in $\boldsymbol{D}$ are per $10^{5} \mu \mathrm{m}^{2}$. Rate of neurosphere growth in $\boldsymbol{B}$ is in $\mu \mathrm{m}^{2} /$ day $\times 10^{3}$. All data are mean \pm SEM; asterisks, $p<0.01$, Student's $t$ test, $n=$ 3/age group.

P21 in these hydrocephalic models (Fig. 2D). Thus, hydrocephalus cannot account for the histogenic defects found in the Foxj1 ${ }^{-1-}$ OB. We therefore postulated that potential Foxj1dependent mechanisms alternative to its established role in ciliogenesis may influence $\mathrm{OB}$ neurogenesis during embryonic and early postnatal periods.

To determine whether or not defects in the Foxj1 ${ }^{-1-} \mathrm{OB}$ were a consequence of disrupted neurogenesis during early development, laminar and cellular integrity in the $\mathrm{OB}$ was assessed during the peak of $\mathrm{OB}$ development between E14.5 and P6. Consistent with our previous report, progenitor activity in the E14.5 Foxj1 ${ }^{-1-}$ LGE and OVZ was relatively unperturbed (Jacquet et al., 2009b). Assessment of structural integrity in the OB at later embryonic and early postnatal time points revealed that the Foxj ${ }^{-1-}$ GL was significantly smaller at E17.5 (normalized GL volume in the Foxj1 ${ }^{-1-} \mathrm{OB}$ was $0.57 \pm 0.12$ of GL volume in wild-type controls; $n=3 ; p<0.05$ ) (Fig. $2 E$ ) and failed to grow between P0 and P6 as the GL in the wild-type OB (Fig. 2F, G). These findings suggested that interrupted neurogenesis rather than degeneration was the likely cause of overall volume deficits in the P21 Foxj1 ${ }^{-1-}$ OB. To further determine whether GL dysgenesis was due to aberrant projections of olfactory receptor axons from the nasal epithelium to the OBs, we obtained sagittal sections of whole heads from P0 Foxj1 $1^{-1-}$ and Foxj1 $1^{+/+}$pups (Fig. $2 F$ ). Olfactory axons positive for the neuronal cell adhesion molecule (NCAM) were clearly present in the P0 Foxj ${ }^{-1-}$ olfactory nerve layer (ONL) (Fig. $2 F$, red arrowheads) and appeared to sprout around the OB (dashed line). Thus, Foxj1 expression did not appear to affect the gross development of olfactory receptor axons or their physical projections to the $\mathrm{OB}$ from the olfactory epithelium. Together, these findings indicated that the $\mathrm{OB}$ defects in the Foxj $1^{-1-}$ brain were due to disrupted neurogenesis during perinatal periods rather than degeneration or hydrocephalus in the Foxj1 deleted brains.

\section{Proliferation and differentiation of neural progenitors in the LGE/SEZ and OVZ/RMS is regulated by Foxj1 expression in vitro}

The severe disruptions in $\mathrm{OB}$ development raised the question of whether or not the defects in the Foxj1 $1^{-1-}$ OB were due to defective progenitor proliferation or differentiation in the LGE/SEZ and OVZ/RMS. As a first approach, we harvested and sorted Foxj1 ${ }^{\text {EGFP }}+$ progenitors from the rostral forebrain of Foxj $1^{-1-\text { EGFP }}$ and Foxj $1^{+/+}$EGFP mice (Fig. $3 A$ ) at P0 and P21, which correspond to two critical time points for $\mathrm{OB}$ histogenesis in our study. EGFP + sorted cells were clonally cultured to generate neurospheres, which arise from clones of neural stem cells and can be propagated to generate neurons and glia upon differentiation. While Foxj $1^{E G F P}+$ sorted progenitors generated large numbers of neurospheres, EGFP+ cells from the Foxj1 ${ }^{-1-}$ forebrain generated significantly fewer neurospheres, and those that began to form were severely retarded in growth at both P0 and P21 (Fig. $3 B, D)$. Moreover, Foxj1 ${ }^{-1-}$ neurospheres seeded on a differentiation-inducing substrate exhibited gliogenic potential (Fig. 3C,D) (GFAP+ astrocytes and $\mathrm{NG}^{2+}$ oligodendrocytes), but their ability to generate neurons was significantly deficient (Fig. 3C, red arrows; $D, \mathrm{TuJ} 1+$ neurons). Together, these results suggest that in the absence of Foxj1-dependent gene expression in the forebrain, proliferation and neurogenic activity of Foxj $1^{\text {EGFP }}+$ progenitors was severely disrupted.

The established diversity among progenitor populations within anatomically distinct domains of the postnatal SEZ and RMS (Pinto and Götz, 2007; Lledo et al., 2008; Alvarez-Buylla et al., 2008) prompted us to microdissect tissues from the ventral SEZ (vSEZ), dorsal SEZ (dSEZ), RMS, and the core of the OB of P7 wild-type and Foxj1 ${ }^{-1-}$ brains (Fig. 4E). Harvested cells were then clonally cultured to form neurospheres as mixed (unsorted) Foxj1 ${ }^{E G F P}+$ and Foxj1 $1^{E G F P}$-negative populations (Fig. $4 A$ ). We found that the dSEZ, RMS, and OB cultured from wild-type 


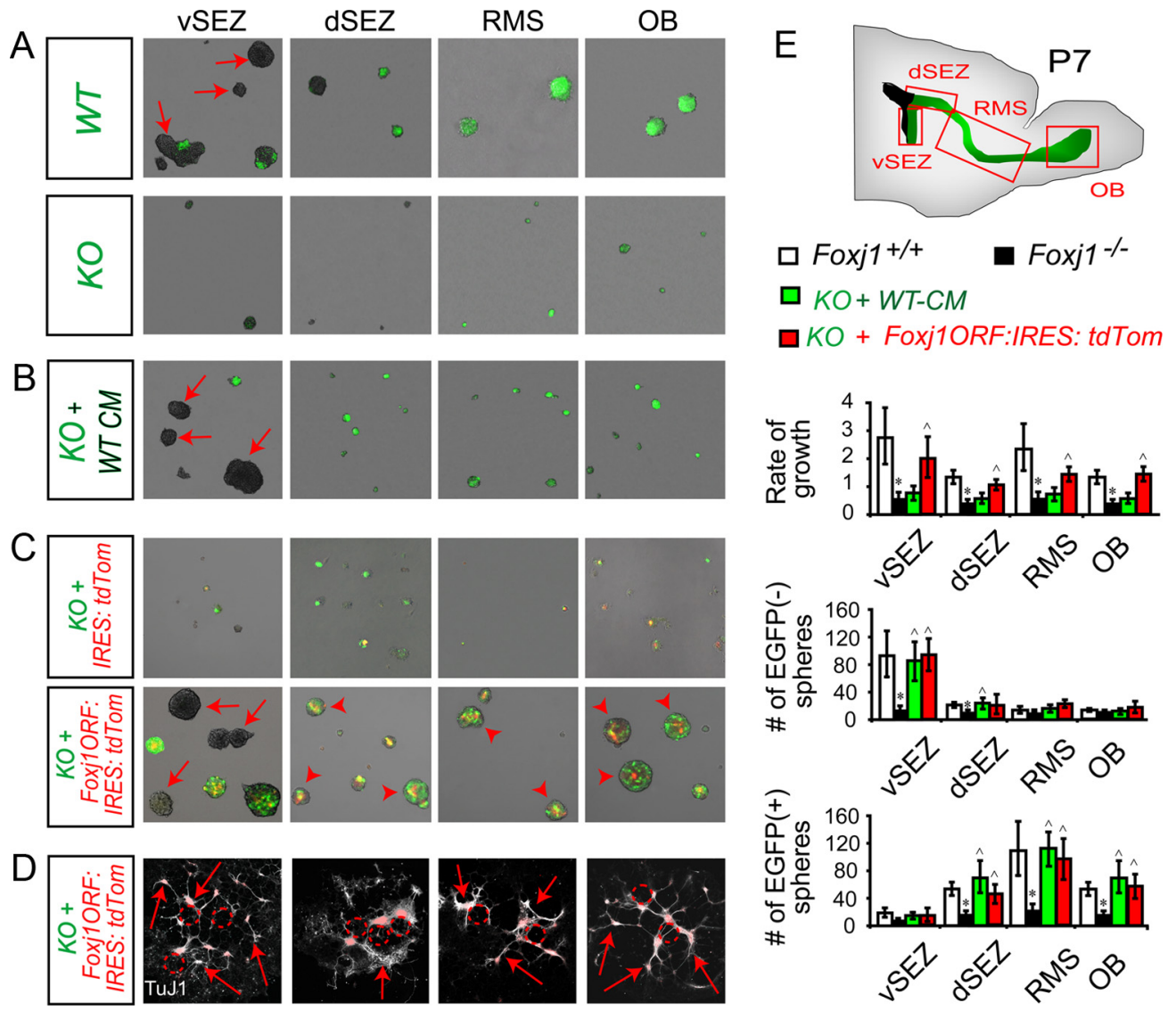

Figure 4. Rescue of the proliferative capacity and differentiation potential of domain-specific neural progenitors in the Foxj $1^{-I-}$ forebrain in vitro. $\boldsymbol{A}$, Representative images of proliferating neurospheres derived from unsorted P7 EGFP+ and EGFP-negative progenitors harvested from Foxj $1^{-1-}$ and Foxj ${ }^{+/+}$VSEZ, dSEZ, RMS, and OB (regions represented as red boxes in $\boldsymbol{E}$ ). $\boldsymbol{B}$, Generation and growth of neurospheres generated from Fox $J 1^{-1-E G F P}$ brains were partially rescued in CM obtained from wild-type spheres (KO + WT-CM). C, FoxJ1 ${ }^{-1-E G F P}$ vSEZ, dSEZ, RMS, and OB cells electroporated in vivo with a construct containing the FoxJ1 ORF and tdTomato under the Foxj1 promoter (KO + FoxJ10RF:IRES:tdTom) or a control IRES:tdTomato coding vector under the Foxj1 promoter (KO + IRES:tdTomato). Cells were harvested $2 \mathrm{~h}$ after electroporation and cultured to generate neurospheres. Forced expression of Foxj1 in Foxj1-null cells rescued their ability to form neurospheres as in wild-type cultures (red arrowheads). $\boldsymbol{D}$. When seeded to differentiate on a laminin/poly-D-lysine substrate, rescued Fox $1^{-I-E G F P}$ neurospheres gained the ability to generate Tuj $1+$ neurons (red arrows). The differentiation zone is defined as the area outside the core of adhered neurospheres (red dotted circle). $\boldsymbol{E}$, Neurospheres in this set of experiments were generated from tissue microdissected from areas delineated by the red boxes labeled VSEZ, ASEZ, RMS, and OB. Number and rate of growth of neurospheres are depicted in the bar charts; asterisks indicate statistically significant difference between Fox $J 1^{+/+}$and Fox $J 1^{-1-}$ neurospheres; the carets ( $\left.{ }^{\wedge}\right)$ indicate statistically significant difference between either KO + WT-CM, or KO + FoxJ10RF:IRES: tdTom and Fox $11^{-1-}$ neurospheres. Numbers for rate of neurosphere growth are $\times 10^{3} \mu \mathrm{m}^{2} / \mathrm{d}$. Numbers of neurospheres are per $10^{5} \mu \mathrm{m}^{2}$. All data are mean \pm SEM; asterisks and ${ }^{\wedge}$ indicate significance, Student's t test, $p<0.01, n=3 /$ region.

brains gave rise to a significantly higher number of Foxj $1^{\text {EGFP }}+$ neurospheres than cells from the vSEZ (Fig. $4 A, E$ ). In contrast, vSEZ cultures included large EGFP-negative neurospheres that were less prevalent in cultures from other domains (Fig. 4A,E).

Strikingly, neurosphere formation in the Foxj1 ${ }^{-1-}$ cultures was significantly retarded in all regions, including the formation of EGFP-negative neurospheres harvested from the vSEZ. To assess whether potential factors in the wild-type cultures could rescue the neurosphere-forming deficits in the Foxj1 ${ }^{-1-}$ cultures, Foxj $1^{-1-}$ cells harvested from each domain were placed in CM obtained from wild-type neurospheres (KO + WT CM) (Fig. $4 B$ ). After $5 \mathrm{~d}$ in vitro, we observed significant growth in the EGFP-negative neurospheres harvested from the vSEZ, while minimal rescue was noted in the number and growth of EGFP+ neurospheres harvested from any domain (Fig. $4 B, E$ ). Thus, factors in CM from Foxj1+ cultures appeared to influence Foxj1-negative progenitors in a nonautonomous manner in vitro.

To investigate whether forced expression of Foxj1 in the SEZ and RMS was sufficient to rescue the Foxj1-null deficits in the proliferation and propagation of neurospheres, we electropo- rated a construct containing the open reading frame of the Foxj1 gene (Foxj1ORF) under the Foxj1 promoter into the anterior ventricles of P2 Foxj1 $1^{-1-\text { EGFP }}$ and Foxj1 ${ }^{+/+ \text {EGFP }}$ mice (Fig. 4C). The construct included an IRES:tdTomato reporter cassette to track electroporated cells ( $p$ Foxj1:Foxj1ORF:IRES:tdTomato). The SEZ, RMS, and OB were harvested $2 \mathrm{~h}$ following electroporation and cultured to generate neurospheres as in the previous experiment. Forced expression of Foxj1 in the Foxj $1^{-1-}$ forebrain resulted in significant rescue of both number and growth rate of Foxj $1^{\text {EGFP }}+$ neurospheres, as well as facilitating the growth of Foxj ${ }^{\text {EGFP }}$-negative spheres in a nonautonomous manner (Fig. $4 C, E)$. Next, we selected Foxj $1^{-1-\mathrm{EGFP}}$ neurospheres with pFoxj1: Foxj1ORF:IRES:tdTomato expression from all domains and seeded them to differentiate. A partial rescue of neurogenic potential in these spheres was revealed following Tuj1 staining (Fig. $4 D$, compare Fig. $3 C$ ). Thus, the loss-of-function and rescue results indicated that Foxj1 expression in Foxj1 + cells is required for their potential function as a subset of forebrain neural progenitors in distinct domains of the SEZ-RMS-OB continuum. Moreover, Foxj $1+$ cells appeared to nonautonomously regulate the proliferation of Foxj1-negative progenitors such as 
those in the ventral domains of the SEZ, a function that is also dependent on Foxj1 expression.

Foxj1 deletion results in aberrant proliferation and differentiation of neural progenitors in the LGE/SEZ and OVZ/RMS in vivo

Based on our in vitro findings, we next asked whether in vivo proliferation of LGE/SEZ and OVZ/RMS progenitors was defective in the Foxj1 $1^{-1-}$ brain during $\mathrm{OB}$ development from E14.5 until P21. Levels of proliferation were quantified using various cell cycle markers including acute BrdU administration to label S-phase cells, Ki67 staining to label cycling cells in the S, G2, or M phases, and labeling for phospho-histone $\mathrm{H} 3$ (PH3) to identify mitotic cells (Fig. 5). We previously showed that progenitor activity in the ventricular and subventricular regions of the LGE was largely unperturbed in the E14.5 embryonic forebrain (Jacquet et al., 2009b). Assessment of proliferation markers at later embryonic stages indicated that a significant depletion in the density of cycling cells emerged in the Foxj $1^{-1-}$ LGE and OVZ beginning $\sim$ E17.5 (Fig. 5). This defect continued to be present in mutant mice at $\mathrm{P} 6$ and $\mathrm{P} 21$, with progressive decline in proliferation indices in the SEZ and RMS of the Foxj1 ${ }^{-1-}$ forebrain relative to their wild-type controls. To determine the extent of autonomous and nonautonomous role of Foxj1 expression on progenitor proliferation, we used Foxj ${ }^{E G F P}$ reporter mice to directly assess the overlap of Foxj1 + and Foxj1-derived cells with proliferating progenitors in vivo. Most cycling cells were EGFP-negative, however, a small fraction of BrdU+ progenitors expressed EGFP in Foxj ${ }^{+/+ \text {EGFP }}$ mice, particularly at E17.5, P0 (data not shown), and P6 (Fig. $5 A, B$ ). The most proliferative population of Foxj1 ${ }^{\text {EGFP }}+$ cells were found in the LGE and OVZ at E17.5, and this proliferation was deficient in the Foxj1 ${ }^{-1-}$ brains confirming our in vitro findings (Fig. 5). Thus, proliferation of $\mathrm{OB}$ progenitors was disrupted beginning in embryonic development and progressively declined during the first 3 weeks of postnatal life.

To further explore whether depleted proliferation in the Foxj $1^{-1-}$ LGE/SEZ and OVZ/RMS affected distinct sets of neural progenitors, BrdU-labeled cells (Fig. 6A, arrows) were characterized by coimmunolabeling for distinct transcription factors marking various progenitor domains (Fig. 6A). In wild-type mice, Foxj $1^{E G F P}+$ cells differentially overlapped with multiple progenitor populations in the LGE/SEZ and OVZ/RMS at E17.5, P6, and P21 (Table 1). The largest overlap was seen at E17.5 in the LGE and OVZ, respectively (Table 1). These cells were confined to a narrow band that corresponded with the pallial-subpallial boundary (Jacquet et al., 2009b). A marked decline in overlap of Foxj ${ }^{\text {EGFP }}$ with the same progenitor cells was noted at P6 and P21 with the exception of cells coexpressing Foxj $1^{E G F P}$ and Tbr2 in the RMS, which peaked at P6 but significantly declined by P21 (Table 1, Fig. 6). At P21, the majority of cycling cells were Foxj1 ${ }^{\text {EGFP }}$ negative. Comparison of Foxj1 $1^{-1-E G F P}$ and Foxj1 $1^{+/+E G F P}$ mice at the same developmental time points revealed that the most pro-

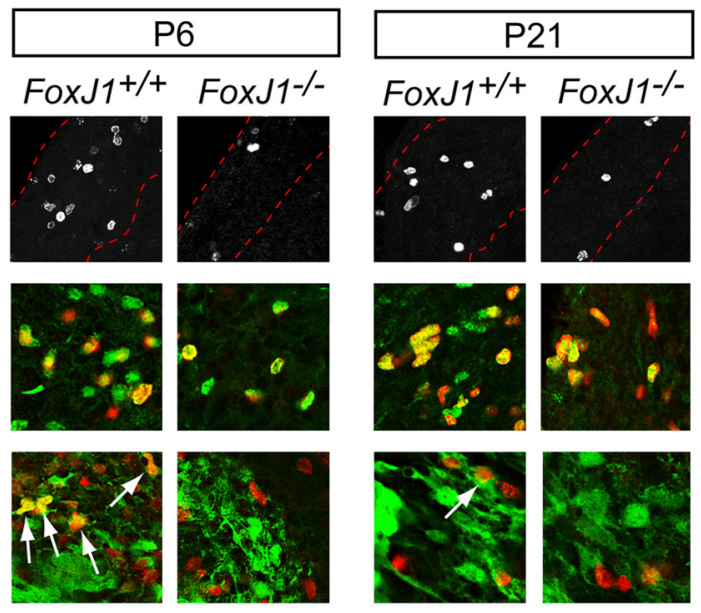

P6

P21
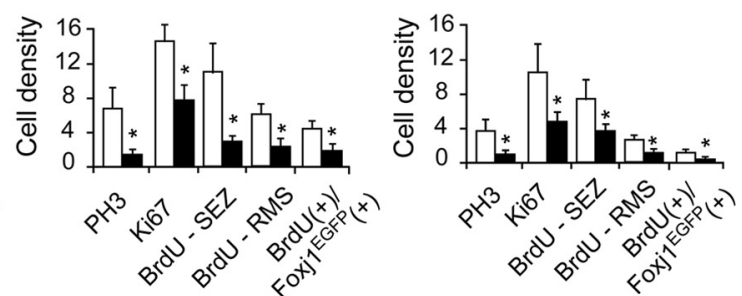

LGE/SEZ and OVZRMS in vivo. A, Confocal photomicrographs of $\mathrm{PH} 3$ and BrdU + /Ki67 + doubled labeled tissue in Foxj $1^{\text {EGFP }}$ brains at various ages (E17.5, P6, and P21). The LGE/SEZ is demarcated by the
red dotted line. B, Stereological estimates of the density of proliferating cells in Foxj ${ }^{+/+}$and Foxj $1^{-/-}$LGE/SEZ and OVZ/RMS. Cell density $=$ number of cells $\times 10^{4} / \mathrm{mm}^{3}$; data are mean \pm SEM; asterisks, Student's $t$ test, $p<0.01 ; n=3$ /age group.

nounced disruption in the Foxj1 $1^{-/-}$stem-cell niche was in the density of Pax6 $+/ \mathrm{BrdU}+$ and $\mathrm{Tbr}^{2+} / \mathrm{BrdU}+$ progenitors at $\mathrm{P} 6$ and P21 (Fig. 6A,B). However, significant alterations were also noted in the density of cycling $\mathrm{Gsx}^{2+}$ and $\mathrm{Dlx}^{2+}$ progenitors in the LGE and the OVZ of E17.5 Foxj1 ${ }^{-1-}$ mice. Together, our in vitro and in vivo findings demonstrate that Foxj1 deletion influenced the proliferation of putative Foxj1 + progenitors cell autonomously, while its regulation of other progenitor types occurred mostly in a cell nonautonomous manner.

\section{In vivo fate-mapping of Foxj1-expressing progenitors}

Our findings thus far indicated that the timed emergence of Foxj1 expression in the wild-type rostral forebrain overlapped with interrupted $\mathrm{OB}$ histogenesis and associated defects in progenitor proliferation in the Foxj ${ }^{-1-}$ LGE/SEZ and OVZ/RMS. Together with our in vitro evidence, these results led us to postulate that Foxj $1+$ cells may directly function as progenitors for $\mathrm{OB}$ neurons at some developmental time point constituting a cellautonomous role for Foxj1+ progenitors in neurogenesis. Simultaneously, Foxj1+ and Foxj1-derived cells appeared to indirectly influence OB-specific progenitor proliferation through potential paracrine activity.

To clearly determine the degree in which Foxj1+ progenitors give rise to neurons in the $\mathrm{OB}$ versus their nonautonomous influence of other progenitor types, we used a genetic fate-mapping approach. Foxj1 ${ }^{\text {Cre::ERT2 }}$ mice (Rawlins et al., 2007) were crossed to multiple reporter lines (Rosa2f floxed-STOPBgal, pCAG floxed-STOP EGFP, $p C A G^{\text {floxed-STOP tdtomato }}$ ) to ensure reliable recombination and reporter activity in different transgenic backgrounds. TAM administration in compound Foxj1 $1_{\text {Cre::ERT2 }}$ /reporter mice resulted in cre-mediated recombination in Foxj1+ cells (Fig. 7). In these 


\section{A}

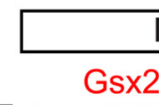

LGE
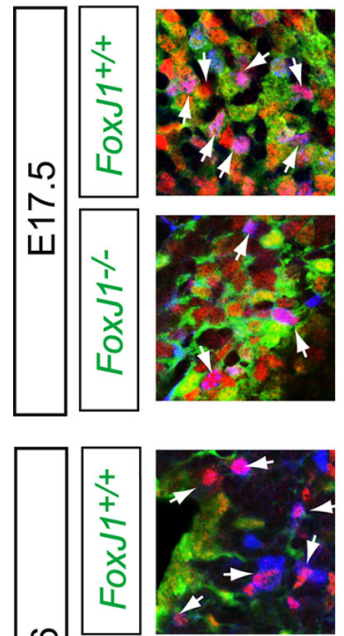

ڤ.
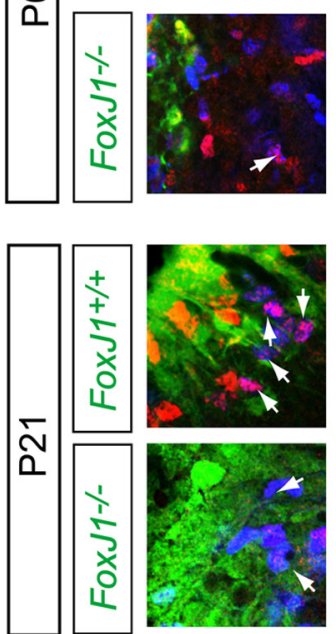

GE/SEZ DIx2
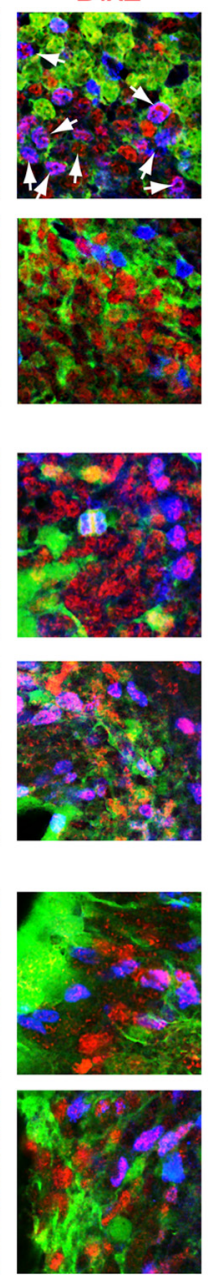

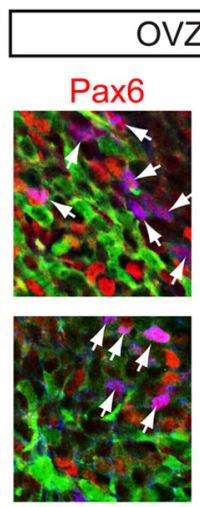

OVZ/RMS
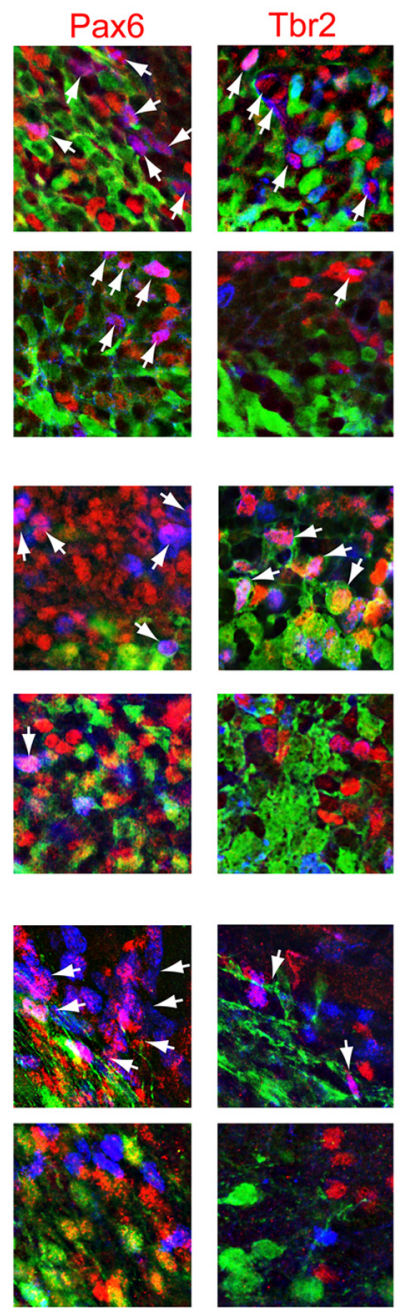

B

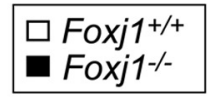

\section{E17.5}

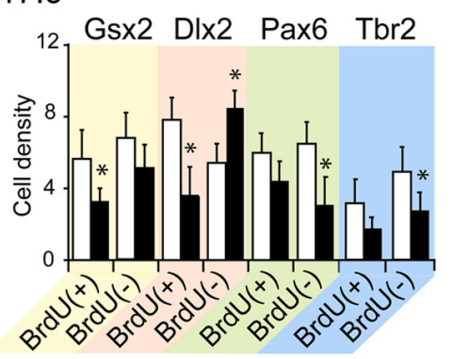

P6

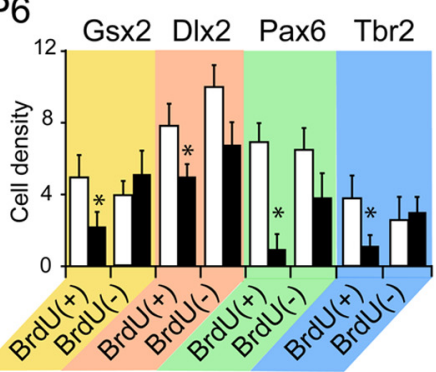

P21

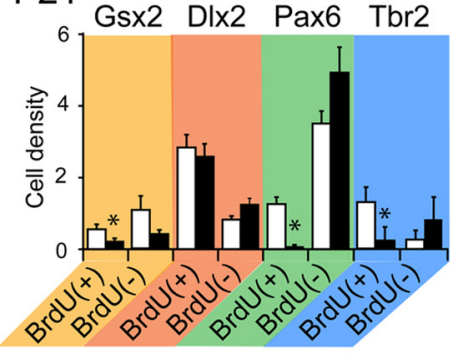

Figure 6. Disrupted proliferation of Gsx2-, Dlx2-, Pax6-, and Tbr2-expressing progenitors in the Foxj $1^{-1-}$ LGE/SEZ and OVZ/RMS in vivo. A, Immunohistochemical labeling for transcription factors unique to distinct progenitor domains in the LGE/SEZ and OVZ/RMS in sagittal brain sections of Foxj $1^{+/+E G F P}$ and Foxj $1^{-1-E G F P}$ mice obtained at E17.5, P6, and P21. BrdU (blue) was labeled following administration as a single pulse $1 \mathrm{~h}$ before death. Antibodies against GsX2, Dlx2, Pax6, and Tbr2 (all red) were used to identify the proliferating fractions in each population (arrows) and their overlap with Foxj $1^{\text {EGFP }}+$ cells (green; also see Table 1). B, Estimates of proliferating and nonproliferating densities of progenitor subtypes were obtained using stereological cell counting. Data are mean \pm SEM; asterisks, $p<0.01$, Student's $t$ test. Cell density $=$ number of cells $\times 10^{4} / \mathrm{mm}^{3} ; n=3$ /age group.

Table 1. Percentage of Foxj $1^{\text {EGFP }}$-positive cells coexpressing markers for various progenitor types in the LGE/SEZ and OVZ/RMS regions at multiple developmental time points

\begin{tabular}{|c|c|c|c|c|c|c|}
\hline Region & Progenitor type & E17.5 & & P6 & & P21 \\
\hline \multirow[t]{2}{*}{ LGE/SEZ } & Gsx2 & $38 \pm 7 \%$ & * & $22 \pm 3 \%$ & * & $9 \pm 2 \%$ \\
\hline & Dlx2 & $17 \pm 4 \%$ & & $13 \pm 1 \%$ & * & $6 \pm 2 \%$ \\
\hline \multirow[t]{2}{*}{ OVZ/RMS } & Pax6 & $21 \pm 4 \%$ & & $18 \pm 5 \%$ & & $13 \pm 2 \%$ \\
\hline & Tbr2 & $14 \pm 3 \%$ & & $22 \pm 8 \%$ & * & $5 \pm 1 \%$ \\
\hline
\end{tabular}

Mean \pm SEM of percentage of Foxj ${ }^{\text {EGFP }}$-positive cells in the LGE/SEZ (GSx2 and DIX2) and OVZ/RMS (Pax6 and Tbr2); $n=3$ per age group. Asterisks indicate statistically significant difference between ages in the expression of each progenitor type, $p<0.01$.

mice, should Foxj1+ cells function as progenitors, then their progeny will continue to express the reporter regardless of whether or not Foxj1 promoter activity continues in the progeny (Fig. 7A). As a positive control and a point of reference we TAMinduced Nestin ${ }^{\text {Cre::ERT2 }}$ mice $\left(N c E^{\text {reporter }}\right.$ ) (Balordi and Fishell, $2007 \mathrm{~b}$ ), based on the established inclusion of Nestin promoteractive progenitors in multiple neuronal lineages in the $\mathrm{OB}$ (Wiese et al., 2004).
To test whether perinatal neurogenesis in the $\mathrm{OB}$ includes the activity of any Foxj1+ progenitors, a single pulse of TAM was administered to $\mathrm{P} 0 \mathrm{Fc} \mathrm{E}^{\beta g a l}$ and $N c E^{\beta g a l}$ mice, and their brains were harvested 3 weeks later at P21 (P21: TAM@P0). $\beta$ gal + cells reporting successful recombination were revealed by immunohistochemistry and were found in the SEZ, RMS, and surprisingly in all OB layers of TAM-induced P2 $F c E^{\beta g a l}$ mice (Fig. 7B). The SEZ, RMS, and OB of $N c E^{\beta g a l}$ mice receiving the same TAM regimen included significantly higher density of $\beta$ gal + cells than $F c E^{\beta g a l}$ brains (Fig. 7 B,D). Cell-specific markers using immunohistochemistry revealed that $\beta g a l+$ cells in the $F c E^{\beta g a l} \mathrm{SEZ}$ were largely ependymal cells $(\mathrm{S} 100 \beta+)$ and a few astrocytes (GFAP +$)$, while in the RMS some of the cells were migrating neuroblasts positive for doublecortin (Dcx) (Fig. 7B). The unexpected presence of Foxj1-derived $\beta$ gal+ cells in the P21 RMS and OB suggested that this discrete population arrived in the OB between $\mathrm{P} 0$ and P21 (Fig. 7B). Comparison with identical inductions in $N c E^{\beta g a l}$ mice indicated that Foxj1-derived cells were only a fraction of the presumptive overall progenitor population in the SEZ-RMS-OB axis. 
Next, we investigated whether the putative Foxj1+ progenitors were developmentally active in the adult-stage neural stem-cell niche in the SEZ and RMS. Single injections of TAM were administered to $\mathrm{P} 21 F_{c} E^{\beta g a l}$ mice and the mice were killed at P60 (P60: TAM@P21). In contrast to P0 TAM inductions, P60: TAM@P21 $F c E^{\beta g a l}$ brains included very few $\beta$ gal + cells in the RMS or OB (Fig. 7C). However, control P60: TAM@P21 $N c E^{\beta g a l}$ brains continued to include substantial densities of $\beta$ gal + cells in the SEZ, RMS, and OB (Fig. 7C,D). Thus, the decline in Foxj1-derived cells in the OB after P21 was independent of age-related decline in postnatal neurogenesis (Wiese et al., 2004).

One concern regarding the above fatemapping results was the efficiency of the TAM-inducible cre-lox system for lineage tracing in our FcE $E^{\beta g a l}$ mice. Using ependymal cells as a point of reference, we found that both $\mathrm{P} 0$ and $\mathrm{P} 21$ inductions resulted in recombination within $23.4 \pm 5 \%$ and $28.8 \pm 4 \%$ of all $\mathrm{S} 100 \beta+$ ependymal cells, respectively ( $n=3$ per age group). This suggested that we may have been underestimating the extent of participation in $\mathrm{OB}$ neurogenesis by Foxj1+ progenitors. To address this issue and to better understand the origin and fate of the Foxj1derived cells, we opted to use mice in which constitutively active crerecombinase is expressed under the Foxj1 promoter (Foxj $1^{\text {cre }}, F c$ ) (Zhang et al., 2007) (Fig. 8A). In these mice, cremediated recombination begins at the onset of Foxj1 expression in the LGE and OVZ, and based on the rate of recombination in the ependymal lineage using the tdTomato reporter (nearly $100 \%$ in $F^{\text {tdtomato }}$ brains at P21 and P90), we presumed it to include the majority of Foxj1+ and Foxj1-derived cells. To conclusively determine whether or not Foxj1+ cells were proliferating in the LGE or OVZ in the P0 brain, we used a Foxj1-specific antibody in combination with $\mathrm{Ki} 67$ and tdTomato (Fig. $8 B$ ). Foxj1+/Ki67+ cycling cells were found within the dorsolateral wall of the LGE and extended into the OVZ (Fig. $8 \mathrm{~B}$, arrows). Interestingly, the levels of tdTomato expression were significantly lower in Foxj1+ progenitors than in their migrating progeny (Fig. $8 \mathrm{~B}$, arrowheads), suggesting a gradual increase in the rate of recombination and reporter expression in the progeny. Importantly, proliferating tdTomato + cells were nearly depleted in Foxj1-null mice on the same fate-mapped background $\left(\right.$ Foxj1 $+/$ Ki67 + cell densities the OVZ: $1.43 \pm 0.3 \times 10^{4} / \mathrm{mm}^{3}$ in WT vs $0.21 \pm 0.08 \times 10^{4} / \mathrm{mm}^{3}$ in KO; $p<0.01$, Student's $t$ test; $n=3)$. Consequently, the overall number of tdTomato + cells in the P0 Foxj1-null OB was significantly reduced $(5.86 \pm 1.3 \times$ $10^{4} / \mathrm{mm}^{3}$ in WT vs $2.06 \pm 1.14 \times 10^{4} / \mathrm{mm}^{3}$ in the KO OVZ; $p<$ 0.01 , Student's $t$ test; $n=3$ ). Together, the fate-mapping experi- ments suggested that Foxj1-derived cells arise from a proliferating population of Foxj1+ progenitors in the LGE and OVZ, and that the expression of Foxj1 is required for the derivation of this progeny.

We next examined the postnatal brain of the constitutively lineage-traced $F c^{\text {tdtomato }}$ mice at P21 and found a significant density of tdTomato + cells in the SEZ, RMS, and OB (Fig. 9A-C). To examine the extent of the Foxj1 lineage, and to determine what portion of the lineage maintained Foxj1 expression, reporter expression was combined with Foxj1 antibody staining. We failed to detect Foxj1 protein in any of the progeny in the OB (data not shown), whereas tdTomato + ependymal cells and a layer of cells in the RMS coexpressed the reporter with Foxj1 (Fig. 9B, arrowheads). Thus, the Foxj1 lineage included both cells that main- 
A
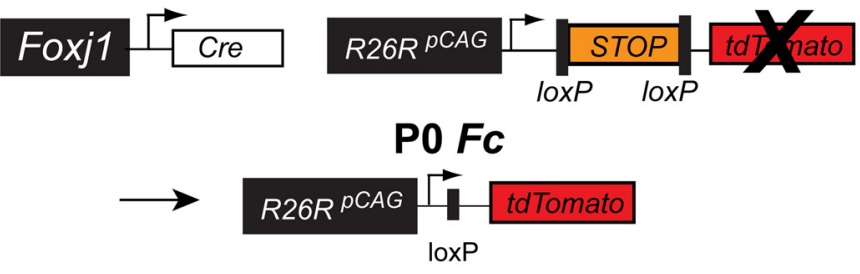

B
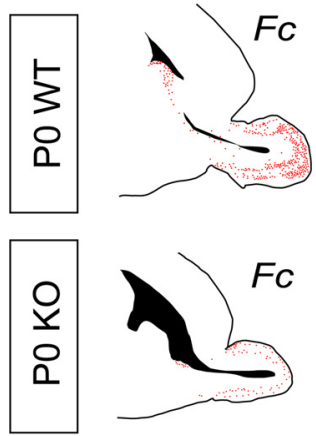

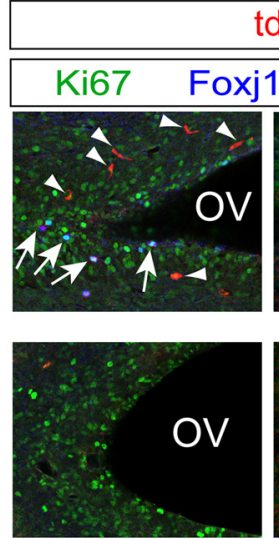

tdTomato
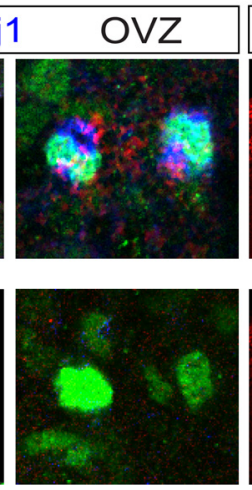

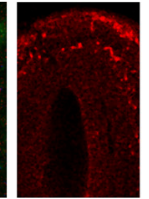

Figure 8. The Foxj1-derived lineage in the $O B$ is dependent on a proliferating population of Foxj1 + progenitors in the $P 0$ OVZ/RMS. A, Constitutive lineage tracing using Foxj $7^{\text {cre }}$ mice crossed to a reporter line expressing tdTomato ( $F(\mathrm{cmice})$. $\boldsymbol{B}$, Diagrams are maps of the distribution of tdTomato + cells in the rostral forebrain of wild-type(WT) and Foxj $1^{-1-}$ (KO) mice at P0. Triple immunohistochemical labeling for Foxj1 (blue), Ki67 (green), and tdTomato (red) in the OVZ of Fc mice on WT and KO backgrounds at P0. Arrows point to Foxj1 + cycling cells; arrowheads point to nearby Foxj1-negative, tdTomato + cells with migratory morphologies. OV, Olfactory ventricle.

tained Foxj1 expression (ependymal cells and ependymal-like cells in the RMS), while the neuronal progeny in the OB were devoid of Foxj1 protein.

Consistent with the progenitor analyses at $\mathrm{P} 0, \mathrm{Fc}^{\text {tdtomato }}$ brains on the Foxj1-null background included significantly lower density of tdTomato + cells in the P21 RMS and OB, clearly demonstrating that Foxj1 expression is essential for generation and placement of Foxj1-derived cells in the OB (Fig. 9B,C). Moreover, as a positive control we administered multiple pulses of TAM on alternating days to $N c E^{\text {tdTomato }}$ mice beginning at $\mathrm{P} 0$ until death at P21 (P21: TAM@P0-P21) (Fig. 9A). Comparison of

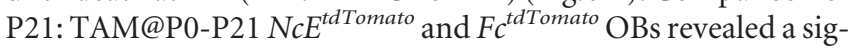
nificantly lower density of tdTomato + cells in the Foxj1 lineage (Fig. 9 B, C). Of all NeuN + neurons in the P21 Fc ${ }^{\text {tdTomato }} \mathrm{OB}$, only $3.4 \pm 1.8 \%$ were tdTomato + , while $12.8 \pm 3.4 \%$ were tdTomato + in the OB of P21: TAM@P0-P21 NcE tdTomato $^{\text {mice }(~} n=3$ per genotype) (Fig. 9D). Finally, to investigate whether or not the Foxj1-derived population was stable over time, we examined wild-type $F c^{\text {tdTomato }}$ mice at P90, when ample time was given for any residual differentiation to complete (Fig. $9 B, C$ ). Only a slight increase in the density of tdTomato + cells in the P90 OB was found compared with the P21 OB in FC mice (1.4 \pm 0.7 -fold increase; $n=3$ per age group), suggesting that the Foxj1-derived population was for the most part stable over early postnatal to young adult period. Moreover, there was little evidence for turnover of the Foxj1-derived population as the RMS of P90 Fc ${ }^{\text {tdTomato }}$ mice included very few tdTomato + cells that were Dcx + or resembled migrating neuroblasts (Fig. 9B,D).

Differences in the neuronal progeny of Nestin and Foxj1 lineages became apparent when we quantified cell types in the $\mathrm{OB}$ expressing tdTomato in $N c E$ and $F c$ lines (Figs. 9C,D, 10). A significant proportion of tdTomato + cells in the OB of both lines of mice were neuronal in their identity (labeled for NeuN or
$\mathrm{TuJ}$, data not shown). Considerable fractions of Nestin-derived cells at P21 in the superficial glomerular and external plexiform layers of the $\mathrm{OB}(\mathrm{GL} / \mathrm{EPL})$ labeled for $\mathrm{CB}(19 \pm 5 \%)$, $\mathrm{TH}(15 \pm 6 \%), \mathrm{CR}$ $(18 \pm 5 \%)$, Tbr2 (17 $\pm 8 \%)$, and Pax6 $(12 \pm 5 \%)$, and some cells in the mitral and granule cell layers (ML/GCL) were positive for Pax6 $(8 \pm 2 \%)$ and CR (15 \pm $7 \% ; n=3$; mean \pm SEM for all data). In contrast, most Foxj1-derived neurons in the GL/EPL or ML/GCL failed to overlap with known neuronal markers (Fig. 10 A), nor did they overlap with the GABAergic populations (data not shown). The only exception was that nearly half of the Foxj1-derived cells in the GL/EPL expressed Tbr1 (49 $\pm 11 \%$ ) (Fig. 10A,C), suggesting that they likely belong to the glutamatergic population (Bulfone et al., 1998; Brill et al., 2009). The subtype proportions of the identified Foxj1-derived cells and the anatomical specificity of their overlap with the Tbr1+ population persisted until P90 (Fig. 10 B, C), suggesting that this population is stable in the adult $\mathrm{OB}$ after its differentiation during embryonic and postnatal periods.

Upon further investigation, we found significant heterogeneity in the overlap of Tbr1 + and Foxj1-derived (tdTomato + ) neurons in individual glomeruli, with no obvious emerging patterns in the dorsal, ventral, or rostral OB sectors (data not shown). Based on a previous study which indicated that most Tbr $1+$ neurons in the OB are derived during embryonic development (Bulfone et al., 1998), we set out to determine the precise timing of the development of the Foxj1-derived Tbr1 + population in the GL/EPL of the OB. First, $F_{c} E^{\beta g a l}$ mice were TAM-induced starting at $\mathrm{P} 0$ and continuing until P21 on alternating days (as in P21: TAM@P0-P21 NcE $E^{t d T o}$. mato inductions) (Fig. 10D). Significantly fewer Foxj1-derived GL neurons $(\beta \mathrm{gal}+)$ were colabeled with Tbrl under this regimen $(11 \%$ of the overall Tbr1+) (Fig. 10D), compared with what was observed in Fc mice. Thus, only a small fraction of Tbr1+ neurons in the GL were derived during early postnatal development.

Second, to determine the embryonic origin of Foxj1-derived neurons in the $\mathrm{OB}$, we conducted BrdU birth-dating experiments. Pregnant females harboring E12.5 and E16.5 Fc ${ }^{\text {tdTomato }}$ embryos were administered BrdU for an entire day through three intraperitoneal injections. Additionally, $F c^{\text {tdTomato }}$ pups at $\mathrm{P} 0$ and P14 were administered the same regimen of $\operatorname{BrdU}$, and all four groups of mice were perfused at P21 (Fig. 11 A). Consistent with our lineage-tracing data, BrdU birth-dating clearly indicated that a significant proportion of the Tbr1+/Foxj1-derived neurons in the GL were generated during embryonic development, suggesting that the birth of the glomerular fraction of the Foxj1 lineage begins during early embryonic periods. However, at no time point could we find larger than $15 \%$ of the Tbr $1+/$ Foxj1-derived population coexpressing BrdU. This was in contrast with the Foxj1-derived subglomerular (largely GCL) population, the birth of which began $\sim$ E16.5 and peaked between P0 and P14 (Fig. $11 A, B)$. Together with the Foxj1-null phenotypes, the fatemapping and birth-dating experiments clearly illustrated that a unique Foxj1-derived lineage is generated during embryonic and 
A

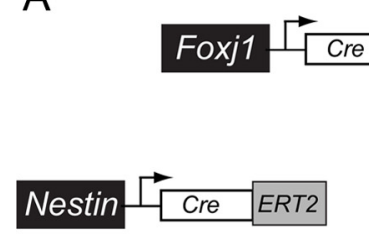

-
$\mathrm{P} 21$ \& P90 FC

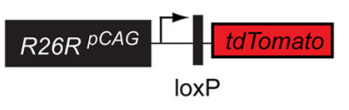

P21 TAM @ P0-P21 NcE
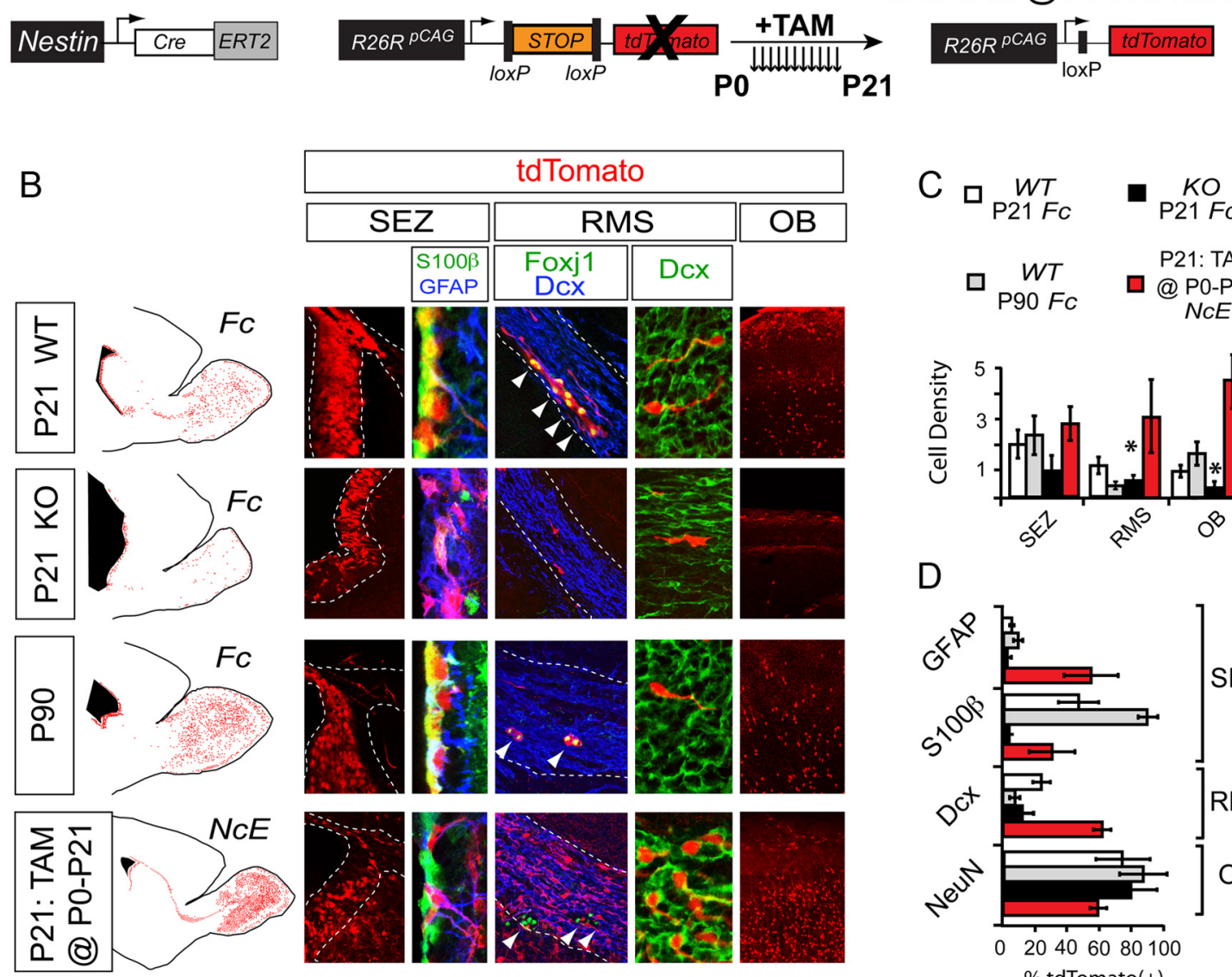
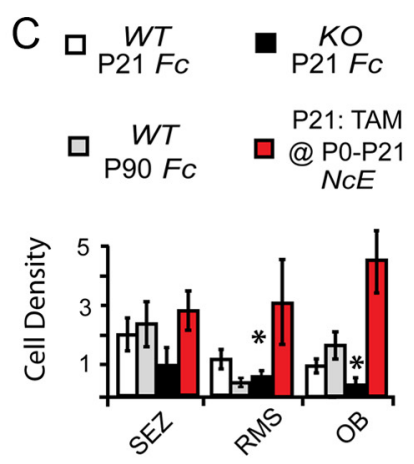

D

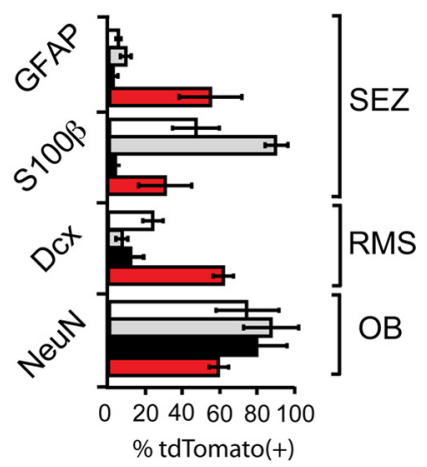

Figure 9. The Foxj1-derived lineage in the OB remains Foxj1 dependent at P21, extends through the SEZ-RMS-OB axis, and is stable overtime. $A$, Constitutive lineage tracing using Foxj ${ }^{\text {cre }}$ mice on the tdTomato reporter system ( $F\left(\right.$ mice). As a positive control, P21 NcE $E^{\text {tdTomato }}$ mice were harvested after receiving TAM injections every $2 \mathrm{~d}$ beginning at P0 until P21 (P21:TAM@P0 -P21). B Cellular and anatomical characterization of lineage-traced tdTomato + cells in the forebrain of Fc and P21:TAM@P0 -P21 NcE mice at the indicated ages and genotypes. Markers:S100 $\beta$, ependymal cells; GFAP, astrocytes; Dcx, neuroblasts. C, Stereological quantification of tdTomato + cells in the SEZ, RMS, and OB of the four groups of mice. Cell density $=$ number of cells $\times 10^{4} / \mathrm{mm}^{3}$. D, Percentage of tdTomato + cells that labeled for various cellular markers in the SEZ, RMS, and OB. Data are mean \pm SEM; asterisks, $p<0.01$, Student's $t$ test; $n=3 /$ genotype.

early postnatal development, and despite its small population, is critical for continued postnatal development in the OB.

\section{Discussion}

In a previous study we demonstrated that Foxj1 expression in the SEZ is required for differentiation of ependymal cells and a small subset of astrocytes that form the postnatal stem-cell niche along the walls of the neostriatum (Jacquet et al., 2009b). Ependymal and astrocytic components of the postnatal stem-cell niche play critical roles in the regulation of progenitor function in the SEZ and RMS, which is essential for neurogenesis in the OBs. Progenitor activity and neuronal migration in the postnatal and adult brain are regulated by factors secreted from ependymal cells and astrocytes (Lim et al., 2000; Kuwabara et al., 2009; Gajera et al., 2010) as well as neuroblasts and progenitors (Nguyen-BaCharvet et al., 2004; Balordi and Fishell, 2007a) via autocrine and paracrine mechanisms. Moreover, beating of motile ependymal cilia filters factors from the CSF and regulates neuronal migration in the RMS (Sawamoto et al., 2006). Our findings in the current study indicate that an ependymal-related lineage with putative paracrine functions extends beyond the ependymal cells that line the lateral ventricular walls. This novel lineage includes a partially unique set of neurons in the $\mathrm{OB}$ that differentiate during embryonic and early postnatal development (Fig. 12).

Foxj1 defines a unique progenitor domain that gives rise to a confined population of $\mathrm{OB}$ neurons

The developmental pattern of Foxj1 expression coupled with the timing of ependymal cell differentiation early in postnatal periods suggests that Foxj1 expression in the embryonic LGE and OVZ may play an alternative function to its canonical role in genesis of motile cilia. We previously found that Foxj1 promoter activity was confined to a narrow band of progenitors that coexpressed Gsx2, Pax6, and Dlx2 at the boundary that separates the telencephalic pallial and subpallial structures (Jacquet et al., 2009b). This suggested that Foxj1 may play a role in a confined population of neural progenitors in addition to its role in generation of motile cilia in ependymal cells. Curiously, we failed to detect robust Foxj1 protein expression within the same progenitor domains before E17.5, with antibody staining or using our own mRNA probes (Jacquet et al., 2009b). Our past findings together with data from the current study suggest that regulation 

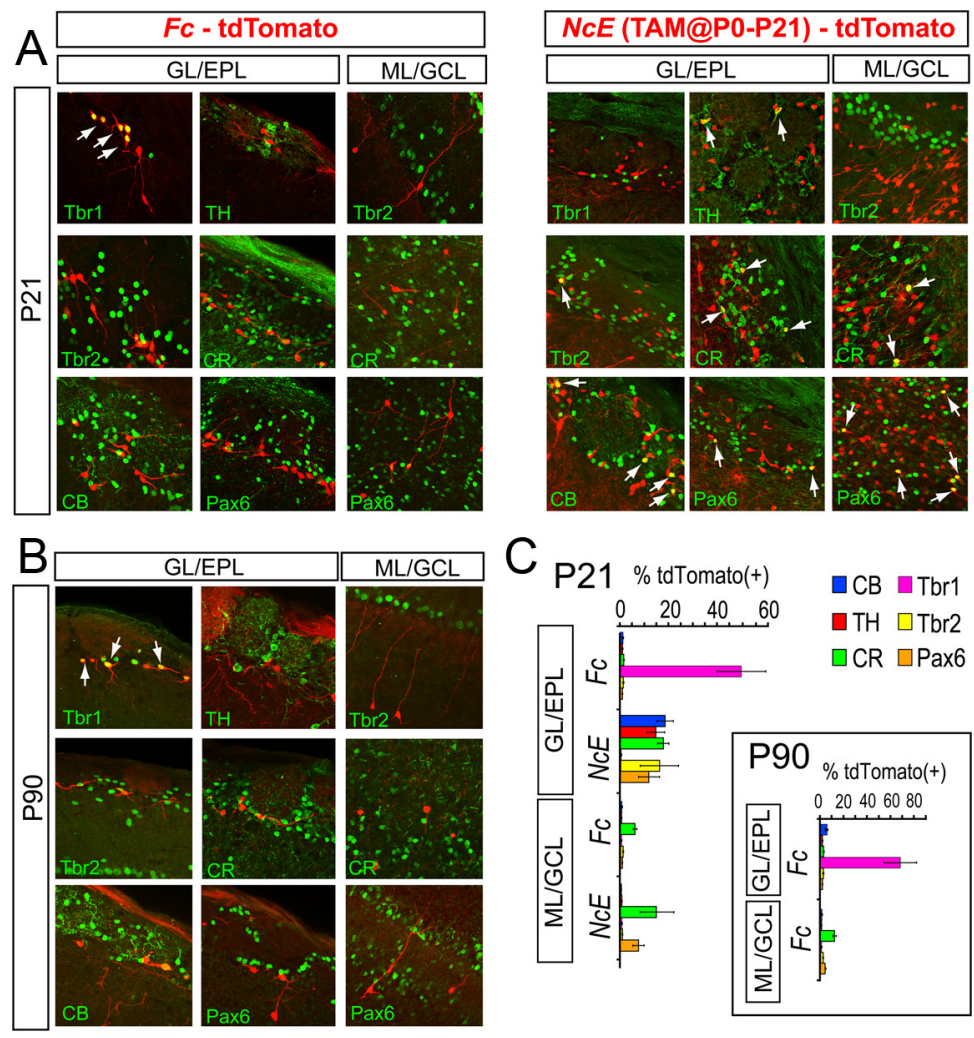

\section{C}
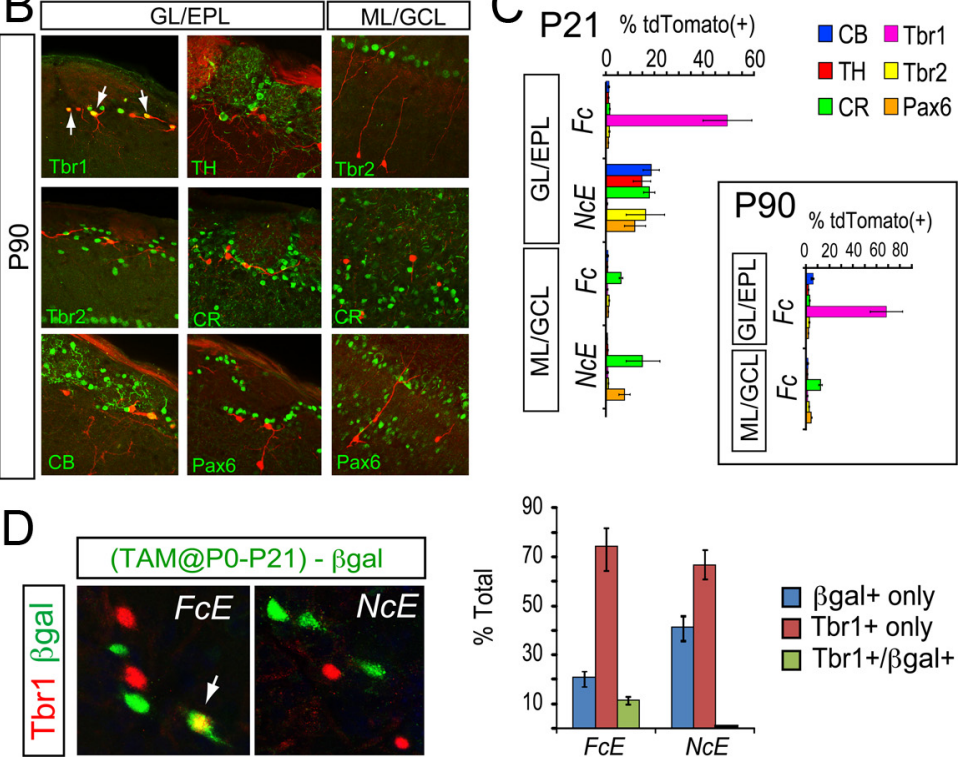

Figure 10. Foxj-derived lineage constitutes a unique neuronal population in the OB. $A$, Characterization of laminarly distinct tdTomato + cell types in the OB of P21 Fc mice and P21 NcE mice (P21:TAM@P0 -P21). B, Identical labeling was conducted in the $\mathrm{P} 90$ OB of $F$ c mice. $C$, Percentage of tdTomato + cells that colabeled for known OB neuronal markers (CR, CB, TH, Tbr1, Tbr2, and Pax6) in the superficial (GL/EPL) and deep (ML/GCL) layers of OB. Data are percentage of reporter + cells \pm SEM; $n=3$ per genotype and age group. D, Comparison of $F C E^{\beta g a l}$ and $N C E^{\beta g a l}$ mice induced from PO until P21. Very few postnatally derived $\beta \mathrm{gal}+$ neurons overlap with the Tbr1 + population in the FCEGL (arrow). Data are percentage of Tbr1 + / $\beta$ gal + double labeled cells over the total number of $\beta \mathrm{gal}+$ and Tbr1 + cells in the GL; data \pm SEM; $n=3$ per genotype.

of activity in the Foxj1 promoter may precede Foxj1 transcription and translation by several days during embryonic development. Repressed expression of Foxj1 during early embryonic stages despite its strong promoter activity would suggest the involvement of complex regulatory mechanisms within the Foxj1 genomic locus that deserve future investigation. Alternatively, it is possible that Foxj1 transcript and protein are expressed in the unique embryonic domain that we have identified in Foxj $1^{\text {EGFP }}$ brains, but at very low and undetectable levels.

Further evidence for alternative functions for Foxj1 expression in the LGE and OVZ became apparent from assessment of the P21 Foxj $1^{-1-} \mathrm{OB}$, which resembles the newborn wild-type $\mathrm{OB}$, suggesting an arrest in growth during perinatal periods. In support of this possibility, there is a profound depletion of neurons in all layers of the Foxj $1^{-/-} \mathrm{OB}$. The most profound defect is the absence of glomeruli as early as E17.5, a time point at which granular and glomerular neurons accelerate their development (for review, see Zou et al., 2009). The glomerular layer never develops during the first three postnatal weeks, suggesting that defects in the Foxj1 $1^{-1-} \mathrm{OB}$ are not because of degeneration, but

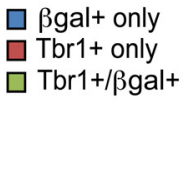

are due to agenesis. These findings led to the hypothesis that Foxj1 expression may regulate progenitor activity during $\mathrm{OB}$ development in vivo.

In vivo fate mapping allowed us to identify a Foxj1-dependent cellular lineage that emerges along the SEZ/RMS/OB axis during prenatal and postnatal development. This cellular population consists of ependymal cells and a small subset of astrocytes in the SEZ and RMS that continue to express Foxj1 (Jacquet et al., 2009b), as well as an unexpected and unique population of neurons in the $\mathrm{OB}$ that are derived from Foxj1+ progenitors but lose their Foxj1 expression. We found that the generation of this unique neuronal population is dependent on Foxj1 expression and is active until the third postnatal week in mice. Quantitative analyses indicate that Foxj1-derived neurons on average account for $\sim 3 \%$ of the estimated total number of neurons in the wild-type P21 OB. However, the Foxj1null defects result in a $65 \%$ reduction of the total population of NeuN+ neurons in the OB. How can the loss of cells that constitutes only $3 \%$ of the total population result in a $65 \%$ reduction in the total population? The only plausible explanation suggests that the few Foxj1-derived cells must influence the development of the population-at-large in a paracrine manner.

\section{Autonomous and nonautonomous regulation of neurogenesis by \\ Foxj1-derived cells}

To address whether and how the Foxj1+ and Foxj1-derived cells regulate overall progenitor activity and $\mathrm{OB}$ neurogenesis, we first examined the developmental potential of Foxj $1+$ progenitors in vitro. Intriguingly, most Foxj1-derived neurospheres are propagated when cells are harvested from the dorsal SEZ (dSEZ), RMS, and OB. In contrast, cells harvested from the ventral domains of the SEZ (vSEZ) generate only a few Foxj1-derived neurospheres but a substantial number of Foxj1-negative neurospheres. Thus, Foxj $1+$ progenitors are situated in a unique domain that extends from the dSEZ through the RMS and into the OB. This domain appears analogous to the pallial/subpallial boundary that was mapped during embryonic development in our earlier study (Jacquet et al., 2009b).

Profoundly, we found that the differentiation potential of Foxj1-derived neurospheres is also dependent on Foxj1 expression. In particular, neurogenic potential of the neurospheres is potently deficient in the few Foxj1-null neurospheres. Loss- and rescue-of-function experiments clearly indicate that neurospheres derived from Foxj1+ progenitors as well as Foxj1negative neurospheres depend on Foxj1 expression for their proliferative and neurogenic potentials. These important findings suggest a cell-autonomous requirement for Foxj1 in the expansion of Foxj1+ neurospheres, and a simultaneous nonau- 
tonomous role for Foxj1+ cells in regulating the expansion of Foxj1-negative neurospheres. In support of a nonautonomous, paracrine, function for Foxj1+ cells, culture media conditioned with Foxj1-expressing neurospheres appear to contain factors that can partially rescue the proliferative potential of Foxj1-null neurospheres. Moreover, reexpression of Foxj1 in Foxj1-null progenitors rescued the expansion and differentiation potential of both Foxj1+ and Foxj1-negative neurospheres. Thus, our current working hypothesis is that Foxj1-dependent secreted factors from Foxj1+ cells influence both the proliferation and differentiation potential of progenitors in the embryonic LGE and OVZ, as well as those in the SEZ, RMS, and $\mathrm{OB}$.

In line with our in vitro findings, progenitor proliferation begins to deteriorate in the embryonic Foxj1-null LGE/OVZ and remains significantly disrupted in the postnatal SEZ and RMS. These defects include disrupted proliferation of known progenitors that express the transcription factors Gsx2, Dlx2, Pax6, and Tbr2, all of which have limited overlap with the Foxj1+ progenitors in the LGE/SEZ and OVZ/RMS. Thus, transcriptional regulation by Foxj1 in a discrete set of cells has a profound nonautonomous effect on overall proliferation within OB-specific progenitor domains during the LGE-to-SEZ conversion. Whether or not this regulation occurs in a Foxj1-dependent manner during adult periods will require conditional genetic approaches to delete Foxj1.

\section{Foxj1-derived cells in the SEZ, RMS, and $O B$}

Our study has identified an apparent physiological system that appears necessary for establishment of the postnatal and adult subependymal stem-cell niche and nonautonomous regulation of $\mathrm{OB}$ neurogenesis. In many ways, Foxj1+ cells and Foxj1-derived neurons appear to function as a perinatal signaling center that is required for the progression and regulation of postnatal neurogenesis in the OB. The mechanisms that drive the development of this system are dependent on the expression of the transcription factor Foxj1.

The glomerular population of Foxj1derived neurons in the OB partially overlaps with the Tbr1+ population, which include tufted glutamatergic neurons. These neurons are known to largely arise during embryonic development (Bulfone et al., 1998). A small fraction of Tbr1+ neurons are either derived or recycled during adult neurogenesis and constitute
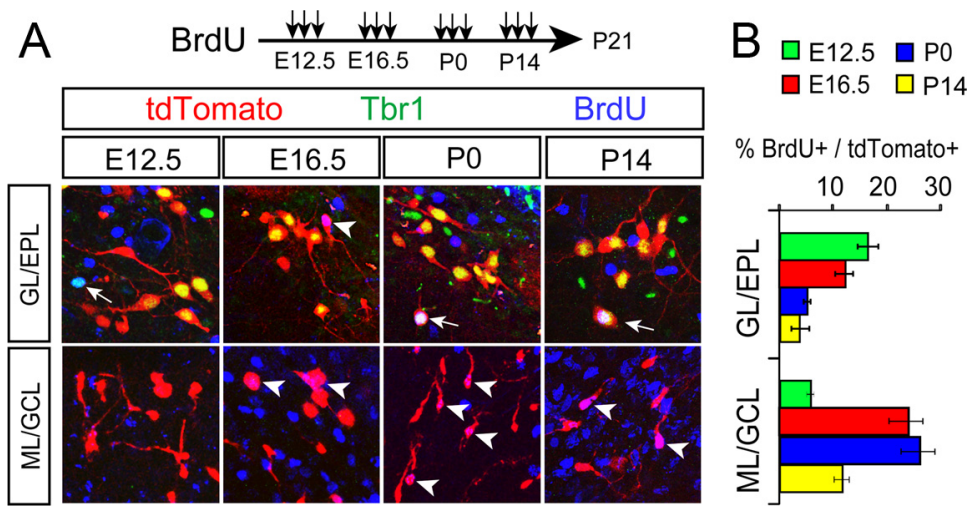

$\% \mathrm{BrdU}+/$ tdTomato+

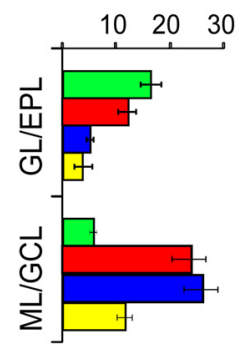

Figure 11. Foxj1-derived neurons in superficial and deep layers of the $O B$ are born during distinct developmental time points. A, BrdU birth-dating was conducted by administering BrdU (3 times, black arrows) to embryonic (E12.5, and E16.5), and postnatal (P0 and P14) Fc mice. Representative high-magnification confocal images of tdTomato + cells and their overlap with BrdU (blue) and Tbr1 (green) labeled cells in the superficial (GL/EPL) and deep (ML/GCL) layers of the OB. Arrows point to Tbr1+/tdTomato +/ BrdU + cells; arrowheads point to tdTomato $+/$ BrdU + cells. $B$, Percentage of BrdU $+/$ tdTomato + cells in the different layers of the $\mathrm{OB}$ in Fc mice that received BrdU pulses at the indicated ages. Data are percentage of reporter + cells $\pm S E M ; n=3 /$ genotype and age group.
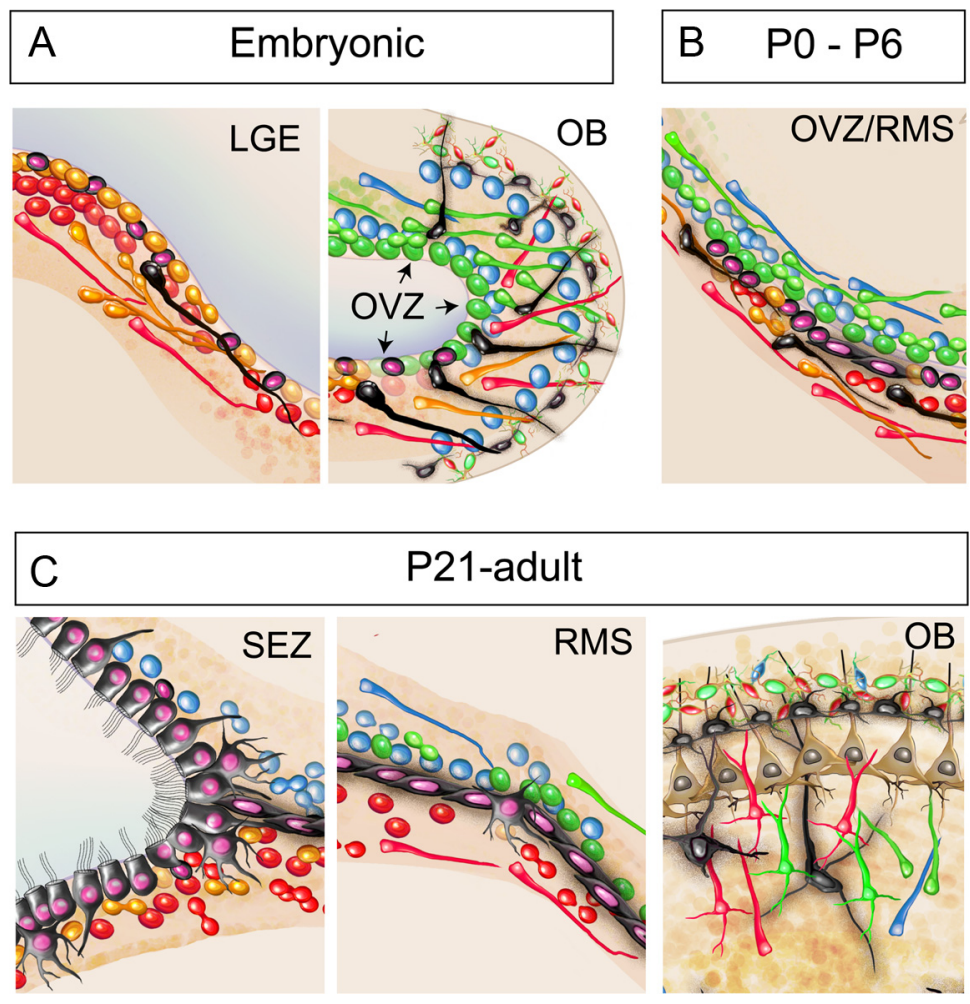

Figure 12. Summary of the temporal development of the Foxj1-dependent lineage in the prenatal LGE-OVZ-OB and postnatal SEZ-RMS-OB axes. Perinatal and adult neurogenesis in the $O B$ depends on proliferation of anatomically distinct progenitors expressing the transcription factors Tbr2 (blue), Pax6 (green), Gsx2 (orange), and Dlx2 (red) and their lineages in the 0B.A, During embryonic development, OB progenitors are located along the ventricular zone of the LGE and OVZ. GsX2 and DIX2 populations are found ventrally along the LGE, while Tbr2 and Pax6 are located on the dorsal aspect of the OVZ. Foxj1 + progenitors (round black cells with pink nuclei) begin their activity during embryonic development and give rise to the glomerular population of Foxj1derived neurons. The migrating progeny of Foxj1+ progenitors lose their Foxj1 expression (black polarized cells without pink nuclei). $\boldsymbol{B}$, In early postnatal development ( $\mathrm{PO}-\mathrm{P6}$ ) the dorsal and ventral aspects of the olfactory ventricles collapse to form the RMS. Foxj1+ progenitors continue to supply the $\mathrm{OB}$ with largely the granule cell layer fraction of Foxj1-derived neurons. $C$, After P21 and into adult periods, Foxj1 + cells (pink nuclei) differentiate into multiciliated ependymal cells and a small population of astrocytes in the SEZ, and a narrow band of epithelial-like cells in the RMS. The neuronal lineage of Foxj1+ progenitors stops propagating after $\mathrm{P} 21$ and constitutes a small population of $\mathrm{OB}$ neurons. Foxj1 specifies this heterogeneous population of cells that includes ependymal cells, a small subset of astrocytes, and a discrete set of neurons in the OB of the adult forebrain. The Foxj1 lineage in this system helps sustain proliferation and differentiation of multiple $0 B$ progenitor types, largely through nonautonomous regulation of their microenvironment. In the absence of Foxj1 expression, the Foxj1-dependent lineage fails to emerge during perinatal development resulting in a profoundly compromised $0 B$ neurogenesis thereafter. 
glutamatergic interneurons juxtaposed in between glomeruli (Brill et al., 2009). The heterogeneity revealed by the partial overlap of Foxj1-derived neurons with the Tbr1 population may prove significant in future identification of their functions; only half of the Foxj1-derived neurons in the glomeruli are Tbr1+ at any given moment, and a significant number of Tbr1 + cells are distinct from the Foxj1-derived population. The birth-dating of Foxj1-derived cells with BrdU presented in this study clearly indicates that the proportion of Tbr $1+$ cells in the OB are largely derived during embryonic development, confirming a previous study (Bulfone et al., 1998). However, Foxj1-derived neurons in the granule cell layer are born later during perinatal development. We failed to identify the Foxj1-derived neurons in the granule cell layer using a battery of known markers for OB neurons, indicating that they are a distinct and previously unappreciated population. Conspicuously, many postnatally born Nestin-derived neurons in the $\mathrm{OB}$ also fail to label with known markers. This observation confirms our previous report using lentiviral-based linage tracing that most adult derived neurons in the $\mathrm{OB}$ may belong to a yet-to-be identified neuronal population (Jacquet et al., 2009a).

\section{Summary}

Our study, for the first time, illustrates that functions of Foxj1dependent cellular differentiation may extend beyond genesis of motile cilia (Stubbs et al., 2008; Yu et al., 2008) and ependymal cell maturation (Jacquet et al., 2009b). Foxj1-dependent gene expression may specify a putative inductive zone that regulates neurogenesis in the perinatal $\mathrm{OB}$. To test whether or not the Foxj1-derived lineage in the $\mathrm{OB}$ is indeed an inductive zone, it will be critical to identity secreted factors that may be specifically expressed by the Foxj1 lineage, and whether or not the expression of such factors is Foxj1-dependent. Identification of a paracrine role for Foxj1-derived cells will be necessary for better understanding the regulation of the transition in embryonic-topostnatal neurogenesis. There is a clear shift in the regulation of neurogenesis during early postnatal weeks, when a radial glialrich niche in the embryo differentiates into an ependymal- and astrocyte-dependent niche. The Foxj1-derived population identified in our study provides a novel molecular, cellular, and physiological context to study the role of this potential inductive cell population that may regulate the transition from embryonic-toadult neurogenesis.

\section{References}

Alvarez-Buylla A, Kohwi M, Nguyen TM, Merkle FT (2008) The heterogeneity of adult neural stem cells and the emerging complexity of their niche. Cold Spring Harb Symp Quant Biol 73:357-365.

Balordi F, Fishell G (2007a) Hedgehog signaling in the subventricular zone is required for both the maintenance of stem cells and the migration of newborn neurons. J Neurosci 27:5936-5947.

Balordi F, Fishell G (2007b) Mosaic removal of hedgehog signaling in the adult SVZ reveals that the residual wild-type stem cells have a limited capacity for self-renewal. J Neurosci 27:14248-14259.

Brill MS, Ninkovic J, Winpenny E, Hodge RD, Ozen I, Yang R, Lepier A, Gascón S, Erdelyi F, Szabo G, Parras C, Guillemot F, Frotscher M, Berninger B, Hevner RF, Raineteau O, Götz M (2009) Adult generation of glutamatergic olfactory bulb interneurons. Nat Neurosci 12:1524-1533.

Bulfone A, Wang F, Hevner R, Anderson S, Cutforth T, Chen S, Meneses J, Pedersen R, Axel R, Rubenstein JL (1998) An olfactory sensory map develops in the absence of normal projection neurons or GABAergic interneurons. Neuron 21:1273-1282.

Doetsch F, García-Verdugo JM, Alvarez-Buylla A (1997) Cellular composition and three-dimensional organization of the subventricular germinal zone in the adult mammalian brain. J Neurosci 17:5046-5061.
Gajera CR, Emich H, Lioubinski O, Christ A, Beckervordersandforth-Bonk R, Yoshikawa K, Bachmann S, Christensen EI, Götz M, Kempermann G, Peterson AS, Willnow TE, Hammes A (2010) LRP2 in ependymal cells regulates BMP signaling in the adult neurogenic niche. J Cell Sci 123:1922-1930.

Gaspard N, Vanderhaeghen P (2010) Mechanisms of neural specification from embryonic stem cells. Curr Opin Neurobiol 20:37-43.

Ghashghaei HT, Weimer JM, Schmid RS, Yokota Y, McCarthy KD, Popko B, Anton ES (2007) Reinduction of ErbB2 in astrocytes promotes radial glial progenitor identity in adult cerebral cortex. Genes Dev 21: $3258-3271$.

Guillemot F (2005) Cellular and molecular control of neurogenesis in the mammalian telencephalon. Curr Opin Cell Biol 17:639-647.

Guillemot F (2007) Cell fate specification in the mammalian telencephalon. Prog Neurobiol 83:37-52.

Hack MA, Saghatelyan A, de Chevigny A, Pfeifer A, Ashery-Padan R, Lledo PM, Götz M (2005) Neuronal fate determinants of adult olfactory bulb neurogenesis. Nat Neurosci 8:865-872.

Hoch RV, Rubenstein JL, Pleasure S (2009) Genes and signaling events that establish regional patterning of the mammalian forebrain. Semin Cell Dev Biol 20:378-386.

Jacquet BV, Patel M, Iyengar M, Liang H, Therit B, Salinas-Mondragon R, Lai C, Olsen JC, Anton ES, Ghashghaei HT (2009a) Analysis of neuronal proliferation, migration and differentiation in the postnatal brain using equine infectious anemia virus-based lentiviral vectors. Gene Ther 16:1021-1033.

Jacquet BV, Salinas-Mondragon R, Liang H, Therit B, Buie JD, Dykstra M, Campbell K, Ostrowski LE, Brody SL, Ghashghaei HT (2009b) FoxJ1dependent gene expression is required for differentiation of radial glia into ependymal cells and a subset of astrocytes in the postnatal brain. Development 136:4021-4031.

Kokovay E, Goderie S, Wang Y, Lotz S, Lin G, Sun Y, Roysam B, Shen Q, Temple S (2010) Adult SVZ lineage cells home to and leave the vascular niche via differential responses to SDF1/CXCR4 signaling. Cell Stem Cell 7:163-173.

Kriegstein A, Alvarez-Buylla A (2009) The glial nature of embryonic and adult neural stem cells. Annu Rev Neurosci 32:149-184.

Kuo CT, Mirzadeh Z, Soriano-Navarro M, Rasin M, Wang D, Shen J, Sestan N, Garcia-Verdugo J, Alvarez-Buylla A, Jan LY, Jan YN (2006) Postnatal deletion of Numb/Numblike reveals repair and remodeling capacity in the subventricular neurogenic niche. Cell 127:1253-1264.

Kuwabara T, Hsieh J, Muotri A, Yeo G, Warashina M, Lie DC, Moore L, Nakashima K, Asashima M, Gage FH (2009) Wnt-mediated activation of NeuroD1 and retro-elements during adult neurogenesis. Nat Neurosci 12:1097-1105.

Lim DA, Tramontin AD, Trevejo JM, Herrera DG, García-Verdugo JM, Alvarez-Buylla A (2000) Noggin antagonizes BMP signaling to create a niche for adult neurogenesis. Neuron 28:713-726.

Lledo PM, Merkle FT, Alvarez-Buylla A (2008) Origin and function of olfactory bulb interneuron diversity. Trends Neurosci 31:392-400.

Merkle FT, Tramontin AD, García-Verdugo JM, Alvarez-Buylla A (2004) Radial glia give rise to adult neural stem cells in the subventricular zone. Proc Natl Acad Sci U S A 101:17528-17532.

Nguyen-Ba-Charvet KT, Picard-Riera N, Tessier-Lavigne M, Baron-Van Evercooren A, Sotelo C, Chédotal A (2004) Multiple roles for slits in the control of cell migration in the rostral migratory stream. J Neurosci 24:1497-1506.

Okano H, Temple S (2009) Cell types to order: temporal specification of CNS stem cells. Curr Opin Neurobiol 19:112-119.

O'Leary DD, Chou SJ, Sahara S (2007) Area patterning of the mammalian cortex. Neuron 56:252-269.

Pinto L, Götz M (2007) Radial glial cell heterogeneity-the source of diverse progeny in the CNS. Prog Neurobiol 83:2-23.

Rawlins EL, Ostrowski LE, Randell SH, Hogan BL (2007) Lung development and repair: contribution of the ciliated lineage. Proc Natl Acad Sci U S A 104:410-417.

Sakano H (2010) Neural map formation in the mouse olfactory system. Neuron 67:530-542.

Sawamoto K, Wichterle H, Gonzalez-Perez O, Cholfin JA, Yamada M, Spassky N, Murcia NS, Garcia-Verdugo JM, Marin O, Rubenstein JL, Tessier-Lavigne M, Okano H, Alvarez-Buylla A (2006) New neurons 
follow the flow of cerebrospinal fluid in the adult brain. Science 311:629-632.

Schmechel DE, Rakic P (1979) A Golgi study of radial glial cells in developing monkey telencephalon: morphogenesis and transformation into astrocytes. Anat Embryol 156:115-152.

Shen Q, Wang Y, Kokovay E, Lin G, Chuang SM, Goderie SK, Roysam B, Temple S (2008) Adult SVZ stem cells lie in a vascular niche: a quantitative analysis of niche cell-cellinteractions. Cell Stem Cell 3:289-300.

Spassky N, Merkle FT, Flames N, Tramontin AD, García-Verdugo JM, Alvarez-Buylla A (2005) Adult ependymal cells are postmitotic and are derived from radial glial cells during embryogenesis. J Neurosci 25:10-18.

Stubbs JL, Oishi I, Izpisúa Belmonte JC, Kintner C (2008) The forkhead protein Foxj1 specifies node-like cilia in Xenopus and zebrafish embryos. Nat Genet 40:1454-1460.
Tavazoie M, Van der Veken L, Silva-Vargas V, Louissaint M, Colonna L, Zaidi B, Garcia-Verdugo JM, Doetsch F (2008) A specialized vascular niche for adult neural stem cells. Cell Stem Cell 3:279-288.

Wiese C, Rolletschek A, Kania G, Blyszczuk P, Tarasov KV, Tarasova Y, Wersto RP, Boheler KR, Wobus AM (2004) Nestin expression-a property of multi-lineage progenitor cells? Cell Mol Life Sci 61:2510-2522.

Yu X, Ng CP, Habacher H, Roy S (2008) Foxj1 transcription factors are master regulators of the motile ciliogenic program. Nat Genet 40: $1445-1453$.

Zhang Y, Huang G, Shornick LP, Roswit WT, Shipley JM, Brody SL, Holtzman MJ (2007) A transgenic FOXJ1-Cre system for gene inactivation in ciliated epithelial cells. Am J Respir Cell Mol Biol 36:515-519.

Zou DJ, Chesler A, Firestein S (2009) How the olfactory bulb got its glomeruli: a just so story? Nat Rev Neurosci 10:611-618. 